\title{
Model Reduction and Stochastic Resonance
}

\author{
P. Imkeller* I. Pavlyukevich ${ }^{\dagger}$
}

14th June 2002

\begin{abstract}
We provide a mathematical underpinning of the physically widely known phenomenon of stochastic resonance, i.e. the optimal noise-induced increase of a dynamical system's sensitivity and ability to amplify small periodic signals. The effect was first discovered in energy-balance models designed for a qualitative understanding of global glacial cycles. More recently, stochastic resonance has been rediscovered in more subtle and realistic simulations interpreting paleoclimatic data: the Dansgaard-Oeschger and Heinrich events. The underlying mathematical model is a diffusion in a periodically changing potential landscape with large forcing period. We study 'optimal tuning of the diffusion trajectories with the deterministic input forcing by means of the spectral power amplification measure. Our results contain a surprise: due to small fluctuations in the potential valley bottoms the diffusion - contrary to physical folklore - does not show tuning patterns corresponding to continuous time Markov chains which describe the reduced motion on the metastable states. This discrepancy can only be avoided for more robust notions of tuning, e.g. spectral amplification after elimination of the small fluctuations.
\end{abstract}

Key words: metastability, stochastic resonance, optimal tuning, spectral power amplification, model reduction, spectral gap, stochastic differential equation, random dynamical system, diffusion, Markov chain, Floquet expansion, double well potential, noise induced transition, climate dynamics

AMS 2000 subject classification: 60H10, 60J60, 60J27, 86A04, 35P15, 37A30, $37 \mathrm{~N} 10$

\section{Introduction and Heuristics}

\subsection{The paradigm of stochastic resonance and heuristics}

To gain physical insight into real climate phenomena or only virtual ones resulting from simulations based on ever more complex general circulation models, reduced models of the climate have been of paramount importance. This fact is nicely exemplified

\footnotetext{
*Institut für Mathematik, Humboldt-Universität zu Berlin, Unter den Linden 6, 10099 Berlin, Germany, email:imkeller@mathematik.hu-berlin.de

${ }^{\dagger}$ Institut für Mathematik, Technische Universität Berlin, Straße des 17. Juni, 136, 10623 Berlin, Germany, email:pavljuke@math.tu-berlin.de
} 
by research performed at the PIK (Potsdamer Institut für Klimafolgenforschung), in particular the group of Rahmstorf (see [21, 37]). Their qualitative results obtained by effective simulations based on climate models of intermediate complexity (CLIMBER) concerning the switching of the thermohaline circulation of the North Atlantic have increased our understanding of mechanisms controlling transitions between warm and ice ages, and more recently even intermediate warmer periods during ice ages (DansgaardOeschger events). They brought back to stage the physical phenomenon of stochastic resonance which had been discovered as a stochastic effect much earlier in the context of very simple and rough energy-balance models designed to explain qualitatively glacial cycles through global energy balances [30, 2, 3, 4].

To explain the physical heuristics and mathematical basics of stochastic resonance, this simple model will be helpful, despite its evident lack of realistic features.

The modern methods of acquiring and interpreting climate records indicate at least seven major climate changes in the last 700,000 years. These changes occurred with the periodicity of about 100,000 years and are characterized by a substantial variation of the average global temperature of about $10 \mathrm{~K}$.

The effect can be explained with the help of a simple energy balance model (for an extended review of the subject see [25]). The Earth is considered as a point in space, and its temporally and spatially averaged temperature $X(t)$ described by the equation

$$
c \dot{X}(t)=E_{\text {in }}-E_{\text {out }} \text {. }
$$

Due to the first law of thermodynamics, the instant change of the Earth's temperature is determined by the difference between the incoming $\left(E_{\text {in }}\right)$ and outgoing $\left(E_{\text {out }}\right)$ radiative energy. The positive constant $c$ describes heat capacity.

$E_{i n}$ is proportional to the so-called solar constant $Q(t)$ which quantifies the solar energy reaching the Earth at time $t$. It is known that due to the influence of Jupiter the eccentricity of the Earth's orbit oscillates with a period of about $10^{5}$ years (Milankovich cycle). This causes the solar constant to vary with the same period. The amplitude of this variation is estimated to be about $0.1 \%$, and we may assume that $Q(t)=$ $Q\left(1+A \sin \left(\frac{2 \pi t}{2 T}\right)\right)$, where $2 T \approx 10^{5}$ years, $A=0.001$.

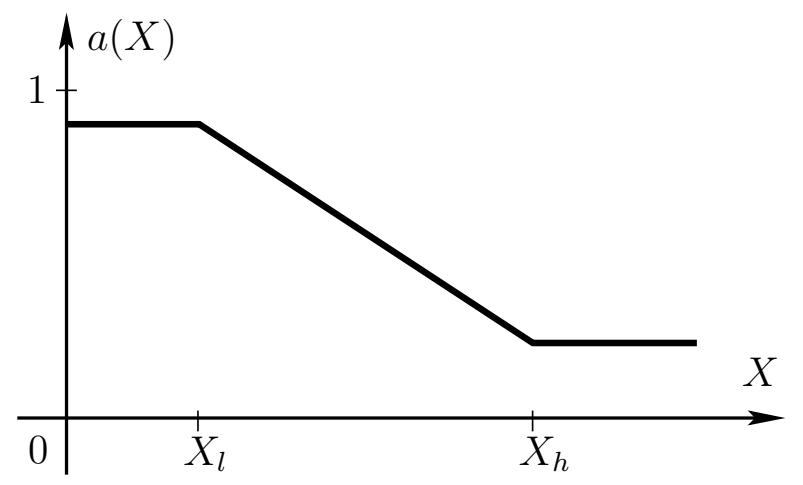

Fig. 1: The Earth's albedo in Budyko-Sellers model as a function of temperature $X$.

On the other hand, $E_{i n}$ is proportional to the global absorption coefficient, which is equal to $1-a(X)$, where $a(X)$ is the average albedo, i.e., the proportion of the solar 
radiation at temperature $X$ reflected back into space. In a simple model suggested by Budyko [11] and Sellers [35] two characteristic temperature levels $X_{l}<X_{h}$ appear. Below $X_{l}$ the surface water is supposed to have frozen, the planet is bright causing high albedo. On the other hand, if $X>X_{h}$, it is very warm, the planet is green and brown, and the albedo is uniformly low. For temperatures between $X_{l}$ and $X_{h}$ a linear interpolation for $a$ is used, see Fig. 1.

To describe $E_{\text {out }}$ the Earth is considered a black body, so that due to the StefanBoltzmann law its radiative energy is proportional to $X^{4}$. So the energy difference takes the form

$$
E_{\text {in }}-E_{\text {out }}=Q\left(1+A \sin \left(\frac{2 \pi t}{2 T}\right)\right)\{1-a(X(t))\}-\gamma X(t)^{4},
$$

where $\gamma$ is the Stefan constant. For appropriate parameter values, the graph of $E_{\text {in }}-$

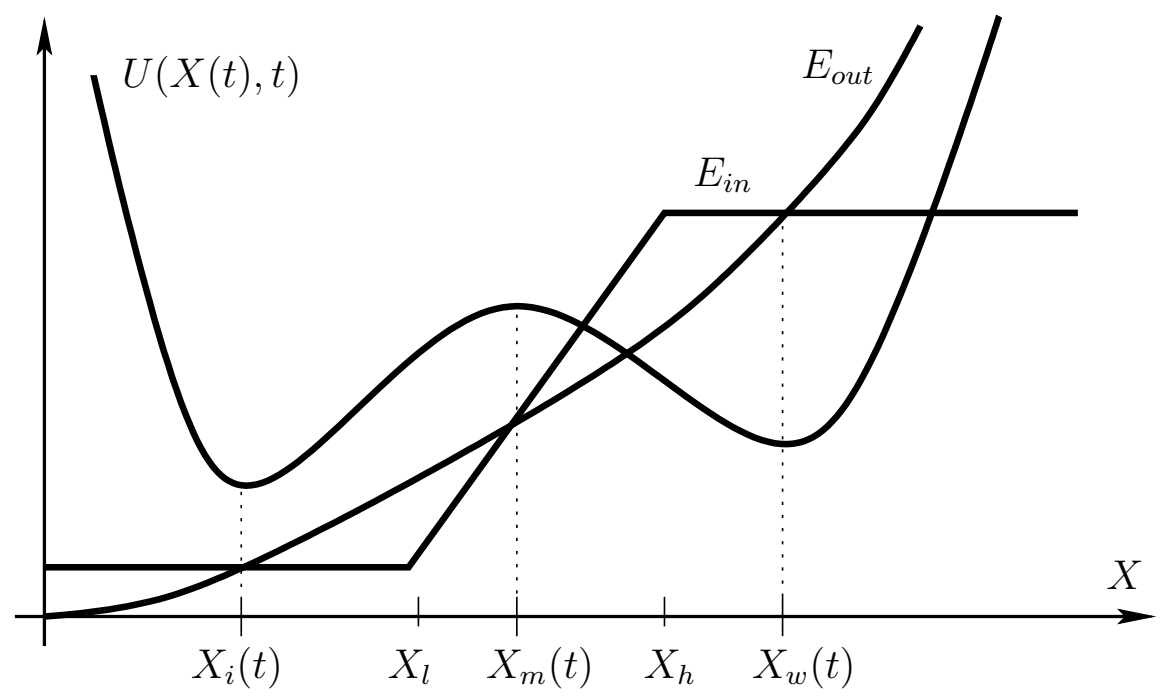

Fig. 2: The energies $E_{\text {in }}$ and $E_{\text {out }}$, and the corresponding potential $U(x, t)$.

$E_{\text {out }}$ has three time-dependent zeroes, say $X_{i}(t), X_{m}(t)$ and $X_{w}(t)$, the stable ones of which can be interpreted as the very slowly varying meta-stable states of the system. As we are in the one-dimensional situation, $\frac{1}{c}\left(E_{\text {in }}-E_{\text {out }}\right)$ can be represented as a gradient of some potential $U(x, t)$, which has two wells with minima in $X_{i}(t)$ and $X_{w}(t)$ and a saddle point at $X_{m}(t)$. This yields the following equation for the global temperature:

$$
\dot{X}(t)=-\frac{\partial}{\partial x} U\left(X(t), \frac{t}{2 T}\right)
$$

The time-dependence of $U(x, t)$ caused by the sin-term from (2) produces two effects. Firstly, the extrema $X_{i}(t), X_{m}(t)$ and $X_{w}(t)$ oscillate deterministically with small amplitudes and a period of about $10^{5}$ years. Secondly, the depths of the potential wells oscillate with the same period. Note that due to the smallness of $A$, the potential does not degenerate into a one-well potential for all $t \geq 0$.

The lower meta-stable state $X_{i}(t)$ is interpreted as the temperature in the Ice Age's regime, the upper state $X_{w}(t)$ in the warm age's. They are both stable, whereas the 
intermediate state $X_{m}(t)$ is not. Any solution of (3) is deterministic and is attracted to one of the meta-stable states. Unfortunately, (3) allows no hopping between $X_{i}$ and $X_{w}$ : the climate of the Earth is completely determined by the initial conditions. Therefore the picture the deterministic system yields can hardly account for the real observations. Both amplitudes of the curves $X_{i}$ and $X_{w}$ are too small to account for the $10 \mathrm{~K}$ observed difference. More importantly, observations show (see e.g. Claussen et al. [12]) that transitions between stable climate states happen very abruptly and spontaneously. In particular, the relaxation time between different states in scales of thousands of years are often only several years.

It is therefore natural that C. Nicolis [30] and Benzi et al. [2, 3, 4] propose to complete the energy-balance equation by a stochastic term of intensity $\varepsilon$, and look at

$$
\dot{X}(t)=-U^{\prime}\left(X(t), \frac{t}{2 T}\right)+\sqrt{\varepsilon} \dot{W}_{t},
$$

where $\dot{W}$ is white noise and $U^{\prime}(x, t)=\frac{\partial}{\partial x} U(x, t)$. So, mathematically we find ourselves in the domain of randomly and periodically perturbed dynamical systems which has not been studied very intensively so far.

Confident to retain the essential effects of periodicity, to simplify further, we allow the time dependence of $U$ to be discontinuous. We consider the asymmetric timeindependent double-well potential $U(x)$ with minima at \pm 1 and a saddle point at 0 . Then, the time-dependent potential is given by $U$ for $t \in\left[0, \frac{1}{2}\right)$, and $U(-\cdot)$ for $t \in\left[\frac{1}{2}, 1\right)$, with a periodic continuation, see Fig. 3. We study the qualitative behaviour of sample paths of (4) for fixed $T$ and different values of $\varepsilon$. To emphasize their dependence on $\varepsilon$ and $T$ we denote by $X_{t}^{\varepsilon, T}(x)$ the solutions of (4) starting at $x$.
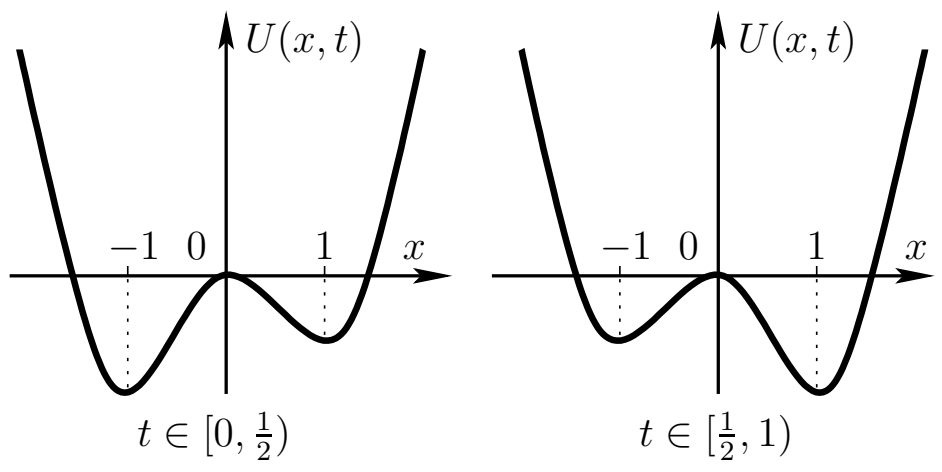

Fig. 3: The time-periodic double-well potential $U(x, t)$ on $t \in[0,1), x \in \mathbb{R}$.

If $\varepsilon$ is very small, jumps between the wells are rare, and the typical time between two jumps is much bigger than $T$. Thus, we observe a picture as in Fig. 4, exhibiting only double-well structure of the potential, not time-periodicity. If $\varepsilon \gg 1$ the jumps occur very often, and the trajectorial variance is so large (see Fig. 5) that we cannot say anything about the spatial geometry of $U$ and its time dependence. The most interesting case concerns moderate $\varepsilon$. It turns out that there exists some 'optimal' value of $\varepsilon$ such that the sample paths become 'as periodic as possible', with period $2 T$. This means that although the trajectories are random, they stay close to the minimum 


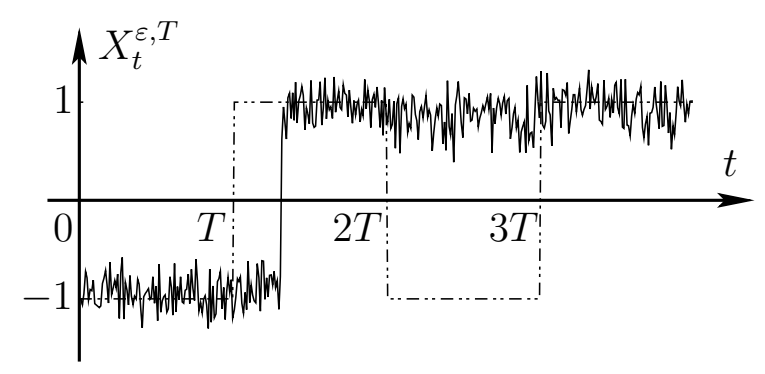

Fig. 4: The sample path of $X^{\varepsilon, T}, \varepsilon \sim 0$.

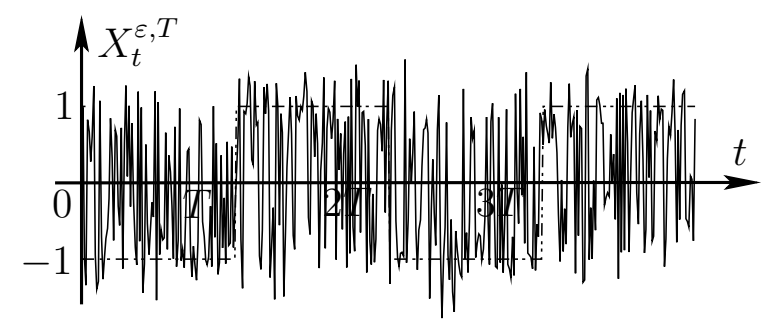

Fig. 5: The sample path of $X^{\varepsilon, T}, \varepsilon \gg 1$.

of the deep well of $U$. As the wells switch their roles, they follow the global minimum of the potential, see Fig. 6. In the language of climate modelling this means that the Ice Ages might be a result of a spontaneous transition or even an 'optimal tuning' of the solar system, that is, the small random fluctuations (of weather, solar activity etc.) and the small deterministic periodic influence of Jupiter might have produced the drastic cumulative effect of abrupt climate change on the Earth. In Fig. 6 this new

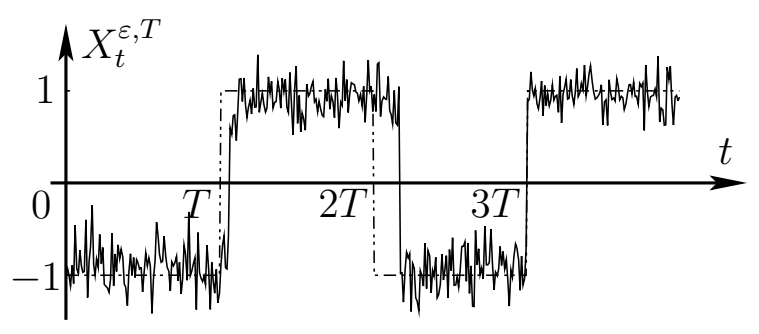

Fig. 6: The sample path of $X^{\varepsilon, T}$ for 'optimal' $\varepsilon$.

regime is shown.

The natural question arises: can one quantify the effect by defining a measure of goodness for the system (4) capable of distinguishing a noise intensity $\varepsilon_{0}$ of optimal tuning for which the trajectories follow the periodic input signal in a 'best possible' way?

Benzi et al. [3] studied the system (4) numerically with respect to the following measure. They considered sample paths of the system on long time intervals, Fourier analyzed them and plotted the power of the spectral component with period $2 T$ as a 
function of $\varepsilon$, averaged over some hundreds of realizations. It turns out that this curve has a strong peak near some $\varepsilon=\varepsilon_{0}$, which means that at this noise level the random trajectories on average have the largest possible periodic component of period $2 T$. This spectral measure of goodness was called the spectral power amplification (SPA) coefficient. The phenomenon of optimal tunability was named stochastic resonance. In comparison to conventional resonance, where the amplitude of the system increases if the frequency of the external periodic driving force is close to the eigenfrequency of the system, stochastic resonance is an effect of amplification of the random output's periodic component as a reply to a weak periodic perturbation in the presence of noise.

Stochastic resonance recently had a comeback in some higher-dimensional systems studied by Ganopolski and Rahmstorf [21] which are much more realistic than energy balance models. In the analysis of the Greenland ice core record, the statistical properties of spontaneous intermediate warmings which are commonly known as Dansgaard-Oeschger events, were found to be consistent with this effect. It is observed that besides the metastable ice and warm age temperature states with transition times around multiples of $10^{4}-10^{5}$ years there is another metastable state at an intermediate temperature accessible from the glacial state. Transition intervals cluster around integer multiples of 1500 years. Ganopolski and Rahmstorf reproduce these observations by a simulation based on the CLIMBER coupled ocean-atmosphere model of moderate complexity established by the Potsdam group. A stability analysis shows the existence of the intermediate metastable state, and suitable small periodic and random excitations of the salinity balance of the North Atlantic as one of the system variables produce temperature curves with abrupt transitions of the observed type. The empirical distribution of the interspike intervals (i.e. multiples of the basic transition period) is seen to be a function of the noise amplitude (see also Imkeller and Herrmann [22]).

Since its discovery in the climate dynamical paradigm sketched above, the effect of stochastic resonance has been found in a variety of physical systems: passive optical bistable systems [13], in experiments with magnetoelastic ribbons [36], in superconducting quantum interference devices [23]. It was also observed in chemical systems [28], as well as in biological ones [34, 26, 19]. For more information the reader is referred to two big reviews on stochastic resonance $[20,1]$ which contain hundreds of references.

While physical studies of stochastic resonance usually deal with real or numerical experiments, mathematical approaches face a number of difficulties. To overcome them, simpler two-state models were suggested in [30, 14, 29]. They reduce the dynamics of a diffusion in a double-well potential to the dynamics of a two-state process on the skeleton space consisting only of the metastable states. For a more general investigation of metastability in stochastic dynamics see Bovier et al. [10, 8, 9]. Indeed, if the noise level is small, the diffusion sample paths are close to the local minima of the potential wells. Thus, if we identify the left and the right wells with, say -1 and 1 , and consider a new process on the discrete space consisting of \pm 1 , it carries information about the inter-well dynamics of the diffusion. The intra-well small fluctuations of the diffusion near the potential minima are now completely neglected.

McNamara and Wiesenfeld in [29] formally reduce the diffusion dynamics to a twostate process. It is known that for small noise intensity $\varepsilon$ transitions between the potential wells occur at Kramers' times. To exponential order they are given by $e^{\frac{\Delta U}{\varepsilon}}$, 
where $\Delta U$ is the work to be done against the potential gradient to cross the barrier. Assuming that transitions of their two-state process are induced by these times and the height of the potential barrier is periodically modulated they approximately determine the spectral properties of the two-state process in the limit as the amplitude of the periodic signal vanishes.

Despite its popularity in the physical society, stochastic resonance attracted the attention of mathematicians relatively lately. The first mathematical paper on this subject by Freidlin [17] considers the phenomenon from the point of view of the FreidlinWentzell theory of large deviations. In this paper diffusions in a general potential landscape with finitely many minima are considered. The attractor basins are subdivided into a hierarchy of cycles with main states corresponding to the deepest one among the cycle states. In the presence of periodic forcing with period time scale $e^{\lambda / \varepsilon}$, in the small noise limit $\varepsilon \rightarrow 0$ transitions between (the main states of) cycles with critical hopping work close to $\lambda$ will be periodically observed. Transitions with smaller critical work may happen, but are negligible in the limit. So in the limit one observes quasi-deterministic periodic hopping between some cycles of potential minima. In the simplest case of two minima of potential depth $\frac{V}{2}$ and $\frac{v}{2}, v<V$, the role of which switches periodically with period $T$, for $T$ larger than $e^{v / \varepsilon}$ it is shown that the diffusion will be close to the deterministic periodic function jumping between the locations of the deepest wells. We shall sketch Freidlin's approach in Section 2.

A different step towards a mathematical understanding of stochastic resonance was made recently by Berglund and Gentz [5, 6, 7]. For parametrized deterministic dynamical systems passing through a pitchfork bifurcation point, the relaxation of solutions to stable equilibria are known to happen after well known delays. Berglund and Gentz exploit this observation to derive pathwise estimates for the trajectories of noisy perturbations of these systems. These results are applied to situations in which the parameter moves the system periodically or in hysteresis loops back and forth through bifurcation points, for example in periodically changing double-well potentials. The papers thus address some questions concerning the trajectorial behaviour in the context of stochastic resonance.

\subsection{Aims and scope of the paper}

This paper deals with a mathematical foundation of the physical paradigm of stochastic resonance. As opposed to Berglund and Gentz [5, 6, 7] we study the physicists' favourite measure of goodness for periodic tuning, the spectral power amplification coefficient (SPA). In particular, we assess the question of whether reduced Markov chain models as in McNamara, Wiesenfeld [29] present a robust and fiable picture of the diffusion's behaviour in the small noise limit.

Our main tool in studying stochastic resonance is the equilibrium (invariant) measure $\mu^{\varepsilon, T}$ of the diffusion given by (4) which lives on the infinite cylinder $\mathbf{R} \times[0,2 T)$. The SPA coefficient we investigate describes the spectral energy of period $2 T$ carried 
by the averaged trajectories and is defined by

$$
\eta^{X}(\varepsilon, T)=\left|\int_{0}^{1} \mathbf{E}_{\mu} X_{2 T s}^{\varepsilon, T} e^{2 \pi i s} d s\right|^{2},
$$

where $\mathbf{E}_{\mu}$ denotes the expectation with respect to the invariant measure. This measure of goodness is considered as a function of the noise level $\varepsilon$ and the half-period $T$. Stochastic resonance occurs if $\varepsilon \mapsto \eta^{X}(\varepsilon, T)$ has a local maximum. We shall determine the optimal tuning rate, i.e. the coordinate $\varepsilon(T)$ of this maximum if $T$ is large. Freidlin's results suggest that we have to restrict our search to the interval $\left(\frac{v}{\log T}, \infty\right)$, since only above the critical level $\frac{v}{\log T}$ the process is able to leave the shallow well at all and therefore show periodic behaviour.

The first step to find optimal tuning intensities $\varepsilon$ consists in effectively reducing the dynamics of the diffusion to the discrete space consisting of the two metastable states. This will be done in Section 3. To mimic well the diffusion dynamics, we define twostate continuous time Markov chains $Y$ with transition probabilities corresponding to the inverses of the Kramers' times for leaving the corresponding wells. So transitions from -1 to 1 happen at rate $\varphi=p e^{-\frac{V}{\varepsilon}}$, and at rate $\psi=q e^{-\frac{v}{\varepsilon}}$ with constant arbitrary pre-factors $p, q$. The time-periodic invariant measure is obtained as a solution of a corresponding forward Kolmogorov equation, and the SPA coefficient of $Y$ described in Proposition 3.3 by

$$
\eta^{Y}(\varepsilon, T)=\frac{4}{\pi^{2}} \frac{T^{2}(\varphi-\psi)^{2}}{(\varphi+\psi)^{2} T^{2}+\pi^{2}}
$$

It is no surprise, that in the large period limit the resonance point exists for all values of the parameters and is located at $\varepsilon(T) \approx \frac{V+v}{2 \log T}$.

In Section 4, we deal with the diffusion. Its equilibrium density is a solution of a forward Kolmogorov (Fokker-Planck) equation, which in this case is a parabolic partial differential equation. We study its solution using the Fourier method of separation of variables. To apply it, the spectral properties of the diffusion's infinitesimal generator have to be studied precisely. This is done in Pavlyukevich [32] and will be dealt with in a forthcoming paper. The essential observations are the following. Besides the trivial eigenvalue of order zero, the first one $\lambda_{1}(\varepsilon)$ turns out to be exponentially small for small noise level $\varepsilon$, and the first eigenfunction's asymptotics can therefore be very well described by means of a series expansion in powers of $\lambda_{1}(\varepsilon)$.

Of central importance to the analysis of goodness of tuning is a spectral gap property of the second eigenvalue. We show that its absolute value is bounded below by some positive constant which is independent of noise intensity. This has crucial implications for $\eta^{X}$ and $\eta^{Y}$ to be developed in Section 4. First of all, it means that only the first two terms in the series expansion of the invariant density matter in the description the SPA coefficient $\eta^{X}$. We obtain the following formula where the remainder term is very small under weak conditions concerning the geometry of $U$

$$
\eta^{X}(\varepsilon, T)=\frac{4}{\pi^{2}}\left(\frac{\int_{\mathbb{R}} y e^{-2 U(y) / \varepsilon}}{\int_{\mathbb{R}} e^{-2 U(y) / \varepsilon}}\right)^{2} \frac{T^{2} \lambda_{1}^{2}}{T^{2} \lambda_{1}^{2}+\pi^{2}}+r(\varepsilon, T) .
$$


The expression for $\eta^{X}$ is close to the SPA coefficient for a two-state Markov chain. Indeed, if we choose the pre-factors in its infinitesimal generator as $p=\frac{\sqrt{U^{\prime \prime}(-1)\left|U^{\prime \prime}(0)\right|}}{2 \pi}$ and $q=\frac{\sqrt{U^{\prime \prime}(1)\left|U^{\prime \prime}(0)\right|}}{2 \pi}$, then $\eta^{X}$ and $\eta^{Y}$ look very similar, since the factor in the parenthesis in (5) is approximately 1 for small $\varepsilon$.

Surprisingly, however, dependencies on the fine structure of the potential function $U$ in the minima beyond the expected curvature properties enter the game and lead to quite unexpected results. For example, a subtle drag away from the other well caused by the sign of the third derivative of $U$ in the deep well suffices to prevent the spectral power amplification curve from having a local maximum in the parameter range suggested by the approximating Markov chain. Contrary to what intuition tells one of the physicists' favourite quality functions does not show resonance effects at all where the reduced model requires it should (Theorem 4.1). The reason for this unexpected dramatic deviation from the approximating Markov chain behaviour lies in the significance attributed to small fluctuations inside the potential wells by the spectral power amplification coefficient. If these fluctuations are cut off, the Markov chain is seen to be a good approximation in the small noise limit, and provides the optimal tuning rate (Theorem 4.2). In all our fine asymptotics studies we extensively use Laplace's method of asymptotic evaluation of integrals depending on a parameter. In an Appendix we collect all facts and formulae about this method used in the paper.

\section{Stochastic Dynamics of Reduced Models}

Although more than four hundred physical papers concerning stochastic resonance were published in the last twenty years, a rigorous mathematical approach to the effect was given only recently in [17]. In this paper stochastic resonance was considered from the point of view of large deviations, and a lower bound for optimal tuning of the random output to the periodic input was given. In this section we briefly formulate the facts needed from Freidlin-Wentzell theory of perturbed dynamical systems [18] in the onedimensional case and discuss the results of [17] concerning the 'optimal tuning' of the double-well oscillator.

\subsection{Diffusion with small noise}

Let $\varepsilon>0$. We first consider the real valued diffusion $X^{\varepsilon}$ in a temporally fixed potential landscape, given by the solution of the SDE

$$
d X_{t}^{\varepsilon}=-U^{\prime}\left(X_{t}^{\varepsilon}\right) d t+\sqrt{\varepsilon} d W_{t}, \quad X_{0}^{\varepsilon}=x, \quad t \geq 0,
$$

where $W$ is a standard Brownian motion, and $U$ a smooth function. We follow Freidlin [17].

For $T>0$, we introduce the action functional on the space $\mathcal{C}[0, T]$ corresponding to $U$ by

$$
S_{0 T}(h)= \begin{cases}\frac{1}{2} \int_{0}^{T}\left(\dot{h}_{s}+U^{\prime}\left(h_{s}\right)\right)^{2} d s, & h \text { absolutely continuous } \\ +\infty, & \text { otherwise }\end{cases}
$$


It is easy to see that $S_{0 T} \geq 0$, and if $S_{0 T}(h)=0$ then $h$ is a trajectory of the dynamical system $\dot{x}=-U^{\prime}(x)$ on the interval $[0, T]$.

Let $x, y \in \mathbb{R}$. By means of the action functional we define the quasipotential

$$
V(x, y)=\inf \left\{S_{0 T}(h): h \in \mathcal{C}[0, T], h_{0}=x, h_{T}=y, T>0\right\} .
$$

The quasipotential describes the work done by a physical particle moving in the potential landscape given by $U$ to get from $x$ to $y$. More precisely, let $\tau_{y}^{\varepsilon}=\inf \{t>0$ : $\left.X_{t}^{\varepsilon}=y\right\}$ and denote by $\mathbf{P}_{x}$ the law of the diffusion starting at $x$. In these terms

$$
V(x, y)=\lim _{T \rightarrow \infty} \lim _{\varepsilon \rightarrow 0}-\log \mathbf{P}_{x}\left(\tau_{y}^{\varepsilon} \leq T\right) .
$$

By means of the quasipotential one can describe the asymptotic behaviour of the diffusion as $\varepsilon \rightarrow 0$. Describing the asymptotics of transition times, it contains a mathematical formulation of Kramers' law.

Theorem 2.1 ([18]) Let $[a, b]$ be a finite interval, $0 \in[a, b]$ be the unique zero of $U^{\prime}(x)$ on the interval, and 0 be the asymptotically stable point of the dynamical system $\dot{x}=-U^{\prime}(x)$. Let $\tau^{\varepsilon}=\inf \left\{t>0: X_{t}^{\varepsilon} \notin[a, b]\right\}$. Then for any $x \in(a, b)$ the following holds:

$$
\begin{aligned}
& \lim _{\varepsilon \rightarrow 0} \varepsilon \log \mathbf{E}_{x} \tau^{\varepsilon}=\min \{V(0, a), V(0, b)\}=V_{0}, \\
& \lim _{\varepsilon \rightarrow 0} \mathbf{P}_{x}\left(e^{\left(V_{0}-\delta\right) / \varepsilon}<\tau^{\varepsilon}<e^{\left(V_{0}+\delta\right) / \varepsilon}\right)=1 \text { for any } \delta>0 .
\end{aligned}
$$

Let us now specify $U$ further. Let it be a double-well potential with minima at \pm 1 and a saddle point at the origin, and assume $U(x) \rightarrow \infty$, as $|x| \rightarrow \infty$. Let $U(-1)=-\frac{V}{2}$, $U(1)=-\frac{v}{2}, U(0)=0,0<v<V$. This implies in particular that -1 marks the deep well.

If $x$ and $y$ are in the same well it is easy to show that

$$
V(x, y)=2 \max \{U(y)-U(x), 0\} .
$$

The pre-factor 2 in (6) explains why we choose the depths as $\frac{V}{2}$ and $\frac{v}{2}$. In particular $U(y)<U(x)$ implies $V(x, y)=0$ because we can descend in the potential landscape from $x$ to $y$ along the deterministic trajectory, on which the action functional equals zero. On the other hand, the way 'up' from $y$ to $x$ costs twice the difference between $U(x)$ and $U(y)$.

Let $x$ and $y$ belong to different wells and suppose for example that $-1 \leq x, y \leq 1$. In this case, we have to overcome a potential barrier of height $U(0)-U(x)$ on the way between $x$ and 0 , and the way 'down' to $y$ is free. Consequently, $V(x, y)=$ $2(U(0)-U(x))$. Analogously, $V(y, x)=2(U(0))-U(y)$. In particular, if $x=-1$ then $V(x, y)=V$, and if $y=1$, we have $V(y, x)=v$. From Theorem 2.1 we can conclude that the mean time to jump from the left well to the right is exponentially large in $\varepsilon$, and is of order $e^{V / \varepsilon}$. The mean time to jump back is smaller and is of order $e^{v / \varepsilon}$. These asymptotics suggest that if we want to record the inter-well motion of the diffusion we should consider it on exponentially long time intervals. The following theorem describes this behaviour precisely. 
Theorem $2.2([\mathbf{1 7}])$ Let $T=T(\varepsilon)$ be such that

$$
\lim _{\varepsilon \rightarrow 0} \varepsilon \log T(\varepsilon)=\lambda>0 .
$$

Let $\delta$ and $A$ be arbitrary positive numbers, $x \in \mathbb{R}$, and $\Lambda$ denote the Lebesgue measure on $\mathbb{R}$. If $\lambda>v$, we have

$$
\Lambda\left(t \in[0, A]:\left|X_{t T(\varepsilon)}^{\varepsilon}+1\right|>\delta\right) \rightarrow 0
$$

in $\mathbf{P}_{x}$-probability as $\varepsilon \rightarrow 0$. If $\lambda<v$, we have

$$
\Lambda\left(t \in[0, A]:\left|X_{t T(\varepsilon)}^{\varepsilon}-\operatorname{sgn} x\right|>\delta\right) \rightarrow 0
$$

in $\mathbf{P}_{x}$-probability as $\varepsilon \rightarrow 0$.

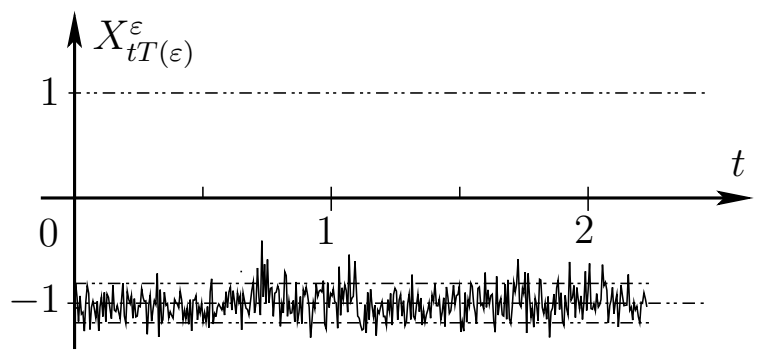

Fig. 7: The sample path of $X_{t T(\varepsilon)}^{\varepsilon}, \lambda>v$.

Indeed, on the time interval of length $e^{\lambda / \varepsilon}$ the diffusion always has enough time to reach the deep well if $\lambda>v$. Moreover, it can make jumps back to the shallow well, but the sum of the periods in which the trajectory is outside of a $\delta$-neighbourhood of -1 has a total length which is negligible in the limit $\varepsilon \rightarrow 0$ (see Fig. 7). If $\lambda<v$ then during the period of length $e^{\lambda / \varepsilon}$ the diffusion does not have enough time to leave the well where it has started, so it stays in the $\delta$-neighbourhood of the corresponding local minimum with high probability.

\subsection{Stochastic periodicity above critical noise level}

Consider now the diffusion $X^{\varepsilon, T}$ in a time periodic potential given by the solution of the SDE

$$
d X_{t}^{\varepsilon, T}=-U^{\prime}\left(X_{t}^{\varepsilon, T}, \frac{t}{2 T}\right) d t+\sqrt{\varepsilon} d W_{t}, \quad X_{0}^{\varepsilon, T}=x \in \mathbb{R}, \quad t \geq 0,
$$

where the potential $U(x, t)=U\left(-x, t+\frac{1}{2}\right)$ is a 1-periodic function of time and $U^{\prime}$ denotes $\frac{\partial U}{\partial x}$. We also assume that on the time interval $\left[0, \frac{1}{2}\right)$ the function $U$ is a doublewell potential as described in the previous section (see Fig. 3, p. 4). The parameter $T$ denotes the half-period with which the drift term in (7) switches its form. On the intervals $[k T,(k+1) T), k=0,1, \ldots$ the diffusion $X^{\varepsilon, T}$ is time-homogeneous, one can calculate the quasipotentials, and if the half period $T$ is long enough, one can apply Theorem 2.2 on each of the intervals. This leads to the following result. 
Theorem 2.3 ([17]) For $\varepsilon>0$, let the half-period $T=T(\varepsilon)$ be such that

$$
\lim _{\varepsilon \rightarrow 0} \varepsilon \log T(\varepsilon)=\lambda>0
$$

and $\delta>0$ and $A>0$ be arbitrary, $x \in \mathbb{R}$. Define a periodic and deterministic function of time by

$$
\phi(t)=\left\{\begin{aligned}
-1, & t \in\left[k, k+\frac{1}{2}\right), \\
1, & t \in\left[k+\frac{1}{2}, k+1\right), \quad k \in \mathbb{Z}_{+},
\end{aligned}\right.
$$

and denote by $\Lambda$ the Lebesgue measure on $\mathbb{R}$. If $\lambda>v$, we have

$$
\Lambda\left(t \in[0, A]:\left|X_{2 T(\varepsilon) t}^{\varepsilon, T}-\phi(t)\right|>\delta\right) \rightarrow 0
$$

in $\mathbf{P}_{x}$-probability as $\varepsilon \rightarrow 0$.

If $\lambda<v$, we have

$$
\Lambda\left(t \in[0, A]:\left|X_{2 T(\varepsilon) t}^{\varepsilon, T}-\operatorname{sgn} x\right|>\delta\right) \rightarrow 0
$$

in $\mathbf{P}_{x}$-probability as $\varepsilon \rightarrow 0$.

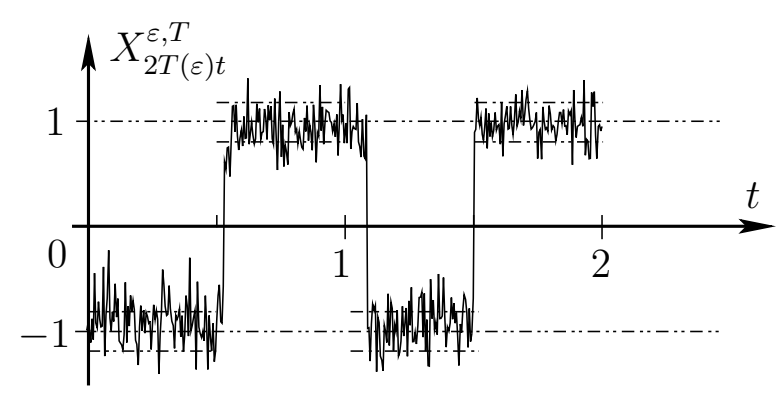

Fig. 8: The sample path of $X_{2 T(\varepsilon) t}^{\varepsilon, T}, \lambda>v$.

The statement of Theorem 2.3 is clear: the function $\phi(t)$ exhibits the location of the global minimum of $U$ at time $2 T(\varepsilon) t$. If the exponential order of $T(\varepsilon)$ is bigger than $v$, then independently of the initial point the trajectory has enough time to reach the deep well during a half-period. In this case we observe some type of stochastic periodicity. If $\lambda<v$, then $T(\varepsilon)$ is too short for the diffusion to 'feel' the change of the potential, and it stays in the same well with very high probability.

Theorem 2.3 provides one possible measure for periodicity of the trajectories. It is given by the Lebesgue measure of the time the diffusion spends outside of a $\delta$-tube of the deterministic function $\phi$. Some type of optimal tuning is also given: if the half-period $T(\varepsilon)$ is such that $\lim _{\varepsilon \rightarrow 0} \varepsilon \log T(\varepsilon)<v$ we observe no reply to the periodic perturbation of the drift in (7). Periodicity in the diffusion paths appears only if $\lim _{\varepsilon \rightarrow 0} \varepsilon \log T(\varepsilon)>v$, and thus we have obtained the lower bound for possible periodic tuning rates. We note also that the lower bound for tuning does not depend on the absolute values of $v$ and $V$. It is only important that $v<V$. 
Theorem 2.3 gives no upper bound for $T(\varepsilon)$. However, the upper bound should exist, but, maybe, for some different measure of quality of the paths. Indeed, let $\lambda \gg v$. The corresponding value $T(\varepsilon)$ is then exponentially larger than both the mean times to jump from the left to the right well and back. This means that $X^{\varepsilon, T}$ can make many excursions to the shallow well, the cumulative duration of which is exponentially small in comparison with the time spent in the deep well. The Lebesgue measure in (8) still goes to zero in probability, but if we look at the sample paths, periodicity is destroyed (see Fig. 9).

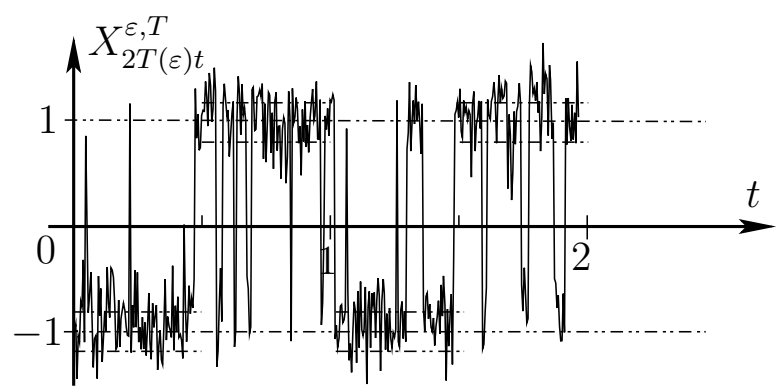

Fig. 9: The sample path of $X_{2 T(\varepsilon) t}^{\varepsilon, T}, \lambda \gg v$.

A natural question arises: what other measures of quality for periodicity of the paths can be considered, and what is the optimal tuning rate they provide? We shall deal with these questions in the next sections, and compare the answers with Theorem 2.3.

\section{Stochastic Resonance in Two-State Markov Chains with Continuous Time}

In the small noise limit, the diffusion given by (4) will spend most of its time very close to the metastable states given by the potential minima \pm 1 , while transitions between the wells happen spontaneously with relatively short relaxation times. It is therefore more than legitimate to expect that the main features of stochastic transition phenomena such as our resonance problem are in fact well described by Markovian motions on the reduced space consisting of the metastable states, with dynamics derived from Kramers' law. This point of view, taken in McNamara, Wiesenfeld [29], is very common in related problems (see Huisinga et al. [24], Fischer et al. [16]). In this section, we shall therefore study the stochastic resonance problem for two state Markov chains with continuous time. With the genuine transition laws to be described in detail below, we shall investigate tuning by means of the physicists' favourite quality measure, the SPA coefficient. It measures the amplitude of the spectral component of the averaged diffusion trajectories corresponding to the input period $2 T$. We shall show that in the simple setting induced by the geometry of the potential of our diffusion both the invariant measure and the SPA coefficient of the reduced Markov chains can be explicitly calculated. As a consequence, the optimal tuning rate maximizing the SPA coefficient as a function of noise intensity $\varepsilon$ can easily be determined. 


\subsection{The Markov chain and its invariant measure}

More precisely, consider a family of Markov chains $Y^{\varepsilon, T}=\left(Y_{t}^{\varepsilon, T}\right)_{t \geq 0}$ on the state space $\mathcal{S}^{Y}=\{-1,1\}$. Let us denote transition probabilities in the following way

$$
p_{i j}(s, t ; \varepsilon, T)=\mathbf{P}\left(Y_{t}^{\varepsilon, T}=j \mid Y_{s}^{\varepsilon, T}=i\right), \quad i, j= \pm 1, \quad 0 \leq s \leq t .
$$

As the diffusion given by (7), the Markov chain $Y^{\varepsilon, T}$ should be time-homogeneous on intervals corresponding to half-periods. On these intervals the behaviour of the diffusion is governed by the potential $U(\cdot)$ or $U(-\cdot)$ (see Fig. 3 ). The spatial antisymmetry of the potential function can be transferred to the Markov chain by setting its infinitesimal generator $Q_{\varepsilon, T}(2 T t)$ to be temporally periodic with period $2 T$ and equal to

$$
Q_{\varepsilon, T}(t)=\left\{\begin{array}{cc}
Q_{1}=\left(\begin{array}{cc}
-\varphi & \varphi \\
\psi & -\psi
\end{array}\right), & 0 \leq t(\bmod 1)<\frac{1}{2}, \\
Q_{2}=\left(\begin{array}{cc}
-\psi & \psi \\
\varphi & -\varphi
\end{array}\right), & \frac{1}{2} \leq t(\bmod 1)<1
\end{array}\right.
$$

We now have to define the transition probabilities $\varphi$ and $\psi$ figuring in $Q_{1}, Q_{2}$ in such a way that the Markov chain retains the dynamical behaviour of the diffusion reduced to its metastable states. In our setting, the states \pm 1 of the process $Y^{\varepsilon, T}$ correspond to the right respectively left well of the potential. Theorem 2.1 states that the mean time to leave the deep well of depth $\frac{V}{2}$ is of order $e^{\frac{V}{\varepsilon}}$ and the mean time to leave the shallow well of depth $\frac{v}{2}<\frac{V}{2}$ is of order $e^{\frac{v}{\varepsilon}}$.

On the other hand, for the time-homogeneous Markov chain governed, for example, by the infinitesimal matrix $Q_{1}$ the mean time to jump from -1 to 1 equals $\varphi^{-1}$ and the mean time to jump from 1 to -1 equals $\psi^{-1}$ which suggests the following expressions for $\varphi$ and $\psi$ :

$$
\begin{aligned}
\varphi=p e^{-V / \varepsilon}, & \psi=q e^{-v / \varepsilon}, \\
\varepsilon>0, & 0<v<V,
\end{aligned}
$$

with some subexponential pre-factors $p, q>0$ not further specified at the moment.

On the intervals $[k T,(k+1) T), k \geq 0$, the process $Y^{\varepsilon, T}$ is therefore time-homogeneous and its transition probabilities (9) are asymptotically as $h \rightarrow 0$ given by

$$
\begin{aligned}
& p_{-1,1}(t, t+h ; \varepsilon, T)=\varphi h+o(h), \\
& p_{1,-1}(t, t+h ; \varepsilon, T)=\psi h+o(h), \quad 0 \leq t(\bmod 2 T)<T,
\end{aligned}
$$

and

$$
\begin{aligned}
& p_{-1,1}(t, t+h ; \varepsilon, T)=\psi h+o(h), \\
& p_{1,-1}(t, t+h ; \varepsilon, T)=\varphi h+o(h), \quad T \leq t(\bmod 2 T)<2 T,
\end{aligned}
$$

To determine the invariant law of the process $Y^{\varepsilon, T}$ we formally have to consider a new two-dimensional Markov process

$$
\mathbf{Y}_{t}^{\varepsilon, T}=\left(Y_{2 T t}^{\varepsilon, T}, \frac{t}{2 T}(\bmod 1)\right), \quad t \geq 0
$$


on the state space $\mathcal{S}^{\mathbf{Y}}=\{-1,1\} \times S^{1}$, which topologically corresponds to the product of two circles.

The process $\mathbf{Y}^{\varepsilon, T}$ is time-homogeneous. Note that we have compressed time: it is convenient to have the time scale independent of the parameter $T$. The infinitesimal generator of $\mathbf{Y}^{\varepsilon, T}$ is given by

$$
B_{\varepsilon, T} f(x, \theta)=\lim _{h \rightarrow 0} \frac{\mathbf{E}_{x, \theta} f\left(Y_{2 T(\theta+h)}^{\varepsilon, T}, \frac{\theta+h}{2 T}(\bmod 1)\right)-f(x, \theta)}{h}, \quad(x, \theta) \in \mathcal{S}^{\mathbf{Y}} .
$$

As $x= \pm 1$ we may think that $B_{\varepsilon, T}$ is defined on the space of vectors $f(\theta)=\left(f^{-}(\theta), f^{+}(\theta)\right)^{*}$ with smooth components. Using (12) and (13) gives

$$
B_{\varepsilon, T} f=\frac{1}{2 T} \frac{d}{d \theta} f+Q_{\varepsilon, T}(\theta) f .
$$

Let the vector $\nu_{\varepsilon, T}=\left(\nu_{\varepsilon, T}^{-}(\theta), \nu_{\varepsilon, T}^{+}(\theta)\right)^{*}, \theta \in[0,1]$, denote the invariant density of $\mathbf{Y}^{\varepsilon, T}$ w.r.t. the product of counting measure on $\{-1,1\}$ and Lebesgue measure on a circle $S^{1}$ normalized so that $\nu_{\varepsilon, T}^{-}(\theta)+\nu_{\varepsilon, T}^{+}(\theta)=1$. We shall call $\nu_{\varepsilon, T}$ the invariant law of the process $Y^{\varepsilon, T}$. Indeed, for $\theta \in[0,1]$

$$
\mathbf{P}_{\nu}\left(Y_{2 T \theta}^{\varepsilon, T}= \pm 1\right)=\nu_{\varepsilon, T}^{ \pm}(\theta) .
$$

The invariant measure satisfies the forward Kolmogorov equation

$$
B_{\varepsilon, T}^{*} \nu_{\varepsilon, T}=0,
$$

and the continuity condition $\nu_{\varepsilon, T}(0)=\nu_{\varepsilon, T}(1)$, where the adjoint operator is given by

$$
B_{\varepsilon, T}^{*} f=-\frac{1}{2 T} \frac{d}{d \theta} f+Q_{\varepsilon, T}^{*}(\theta) f .
$$

From the symmetry between $Q_{1}$ and $Q_{2}$ in (10) we deduce

Proposition 3.1 The invariant measure of the process $\mathbf{Y}^{\varepsilon, T}$ has the following symmetry property: $\nu_{\varepsilon, T}^{ \pm}(\theta)=\nu_{\varepsilon, T}^{\mp}\left(\theta+\frac{1}{2}\right), 0 \leq \theta \leq \frac{1}{2}$.

Proof: The statement follows easily from the fact that if, for example, $\nu=\left(\nu^{-}, \nu^{+}\right)^{*}$ is a solution of $-\frac{1}{2 T} \dot{\nu}+Q_{1}^{*} \nu=0$, then $\bar{\nu}=\left(\nu^{+}, \nu^{-}\right)^{*}$ satisfies $-\frac{1}{2 T} \dot{\bar{\nu}}+Q_{2}^{*} \bar{\nu}=0$, together with the continuity condition and the uniqueness of the invariant measure. Here, $\dot{\nu}=\frac{d}{d \theta} \nu$.

It follows from Proposition 3.1 that in order to find the invariant measure, it is enough to solve the boundary value problem

$$
\left\{\begin{array}{l}
-\frac{1}{2 T} \frac{d}{d \theta} \nu_{\varepsilon, T}+Q_{1}^{*} \nu_{\varepsilon, T}=0 \\
\nu_{\varepsilon, T}^{-}(0)=\nu_{\varepsilon, T}^{+}\left(\frac{1}{2}\right) \\
\nu_{\varepsilon, T}^{-}(\theta)+\nu_{\varepsilon, T}^{+}(\theta)=1 \\
\nu_{\varepsilon, T}^{ \pm}(\theta)>0, \quad \theta \in\left[0, \frac{1}{2}\right]
\end{array}\right.
$$

which is done in the following 
Proposition 3.2 For $T>0$ and $\varphi$ and $\psi$ defined by (11), the invariant measure of the process $Y^{\varepsilon, T}$ equals

$$
\begin{aligned}
& \left\{\begin{array}{l}
\nu_{\varepsilon, T}^{-}(\theta)=\frac{\psi}{\varphi+\psi}+\frac{\varphi-\psi}{\varphi+\psi} \frac{e^{-2(\varphi+\psi) T \theta}}{1+e^{-(\varphi+\psi) T}} \\
\nu_{\varepsilon, T}^{+}(\theta)=\frac{\varphi}{\varphi+\psi}-\frac{\varphi-\psi}{\varphi+\psi} \frac{e^{-2(\varphi+\psi) T \theta}}{1+e^{-(\varphi+\psi) T}}
\end{array}\right. \\
& \left\{\begin{array}{l}
\nu_{\varepsilon, T}^{-}\left(\theta+\frac{1}{2}\right)=\nu_{\varepsilon, T}^{+}(\theta), \\
\nu_{\varepsilon, T}^{+}\left(\theta+\frac{1}{2}\right)=\nu_{\varepsilon, T}^{-}(\theta), \quad 0 \leq \theta \leq \frac{1}{2}
\end{array}\right.
\end{aligned}
$$

Proof: Solving the differential equation in (15) and using the normalizing condition gives the following general solution

$$
\nu_{\varepsilon, T}^{-}(\theta)=\frac{\psi}{\varphi+\psi}+A e^{-2(\varphi+\psi) T \theta}, \quad \nu_{\varepsilon, T}^{+}(\theta)=\frac{\varphi}{\varphi+\psi}-A e^{-2(\varphi+\psi) T \theta},
$$

where $A$ is an arbitrary constant. Applying the boundary condition leads to

$$
A=\frac{\varphi-\psi}{\varphi+\psi} \frac{1}{1+e^{-(\varphi+\psi) T}}
$$

which concludes the proof.

Note that $\nu_{\varepsilon, T}^{ \pm}(\theta)$ is a sum of two parts. For $\theta \in\left[0, \frac{1}{2}\right]$, the time-independent pair $\left(\frac{\psi}{\varphi+\psi}, \frac{\varphi}{\varphi+\psi}\right)$ is the invariant measure of the time-homogeneous Markov chain with infinitesimal generator $Q_{1}$, and $\left(\nu_{\varepsilon, T}^{-}\left(\frac{1}{2}\right), \nu_{\varepsilon, T}^{+}\left(\frac{1}{2}\right)\right) \rightarrow\left(\frac{\psi}{\varphi+\psi}, \frac{\varphi}{\varphi+\psi}\right)$ exponentially fast with rate $\varphi+\psi$ as $T \rightarrow \infty$. This is an illustration of a classical result about convergence of the law of a stochastic process to its invariant law.

On the second half-period, the Markov chain is governed by the infinitesimal generator $Q_{2}$ and therefore $\left(\nu_{\varepsilon, T}^{-}(1), \nu_{\varepsilon, T}^{+}(1)\right) \rightarrow\left(\frac{\varphi}{\varphi+\psi}, \frac{\psi}{\varphi+\psi}\right), T \rightarrow \infty$, which is the invariant law of the Markov process with generator $Q_{2}$.

The invariant measure obtained in Proposition 3.2 is our main tool in studying the periodic properties of the process $Y^{\varepsilon, T}$. In the following subsection we shall introduce and study the physical measure of goodness of periodic tuning.

\subsection{Spectral power amplification coefficient}

We define the Spectral Power Amplification coefficient as the power carried by the averaged trajectory of the Markov chain on the period $2 T$ by

$$
\eta^{Y}(\varepsilon, T)=\left|\int_{0}^{1} \mathbf{E}_{\nu} Y_{2 T s}^{\varepsilon, T} e^{2 \pi i s} d s\right|^{2},
$$

where $\mathbf{E}_{\nu}$ denotes the expectation w.r.t. the invariant measure $\nu_{\varepsilon, T}$.

The goal of this section is to study the behaviour of the SPA coefficient as a function of $\varepsilon$ and $T$, in particular its local maximum as a function of $\varepsilon$ for fixed large $T$. 
Proposition 3.3 The SPA coefficient is given by

$$
\eta^{Y}(\varepsilon, T)=\frac{4}{\pi^{2}} \frac{T^{2}(\varphi-\psi)^{2}}{(\varphi+\psi)^{2} T^{2}+\pi^{2}}
$$

with $\varphi$ and $\psi$ defined by (11).

Proof: We use (16) to obtain

$$
\begin{aligned}
& \int_{0}^{1} \mathbf{E}_{\nu} Y_{2 T s}^{\varepsilon, T} e^{2 \pi i s} d s=\int_{0}^{1}\left(\nu_{\varepsilon, T}^{+}(s)-\nu_{\varepsilon, T}^{-}(s)\right) e^{2 \pi i s} d s \\
& =2 \int_{0}^{\frac{1}{2}}\left(\nu_{\varepsilon, T}^{+}(s)-\nu_{\varepsilon, T}^{-}(s)\right) e^{2 \pi i s} d s=2 \frac{\varphi-\psi}{\varphi+\psi}\left(\frac{i}{\pi}+\frac{1}{\pi i-(\varphi+\psi) T}\right) .
\end{aligned}
$$

This directly leads to (18).

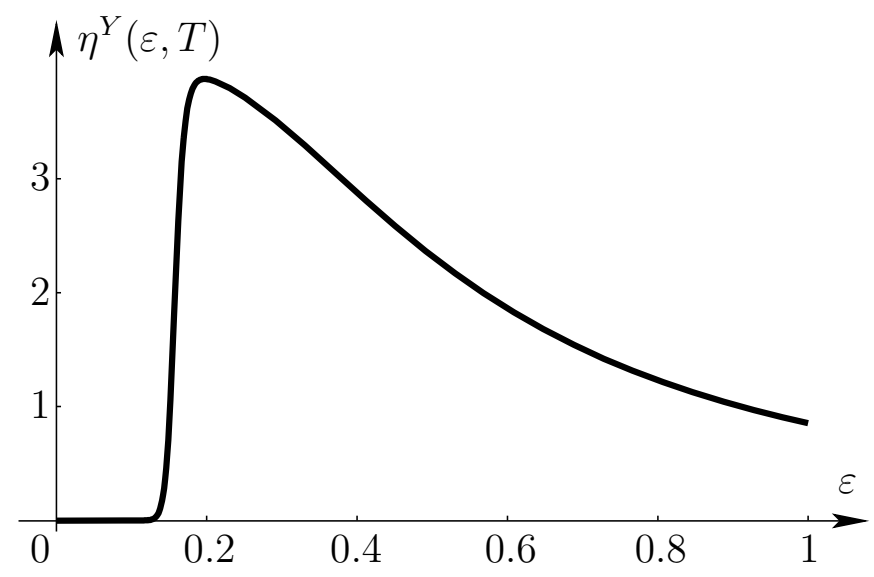

Fig. 10: If $p=q=1, V=3, v=2$ and $T=10^{6}$ the SPA coefficient $\eta^{Y}(\varepsilon, T)$ has a local maximum at $\varepsilon \approx 0.197$.

In the following Proposition we describe the local maxima of $\varepsilon \mapsto \eta^{Y}(\varepsilon, T)$.

Proposition 3.4 a) We have $\eta^{Y}(\varepsilon, T) \geq 0, \eta^{Y}(0, T)=0$.

b) Let $0<\beta=\frac{v}{V}<1$ and $T>0$ be fixed. Consider the function

$$
\widehat{p}(q)=\frac{2 \pi^{2} q \beta}{2 q^{2} T^{2}(1-\beta)+\pi^{2}+\sqrt{\left(2 q^{2} T^{2}(1-\beta)+\pi^{2}\right)^{2}+8 \pi^{2} q^{2} T^{2} \beta(1-\beta)}},
$$

for which $0 \leq \widehat{p}(q) \leq \beta q$, if $q>0$, and $\widehat{p}^{\prime}(0)=\beta$.

Let us consider three disjoint domains (see Fig. 11)

$$
\begin{aligned}
& U_{0}=\{(p, q): 0<p \leq \widehat{p}(q), q>0\}, \\
& U_{1}=\{(p, q): \widehat{p}(q)<p \leq q, q>0\}, \\
& U_{2}=\{(p, q): 0<q<p\}
\end{aligned}
$$

Then the SPA coefficient $\eta^{Y}(\varepsilon, T)$ depends in the following way on the pre-factors $p$ and $q$ : 


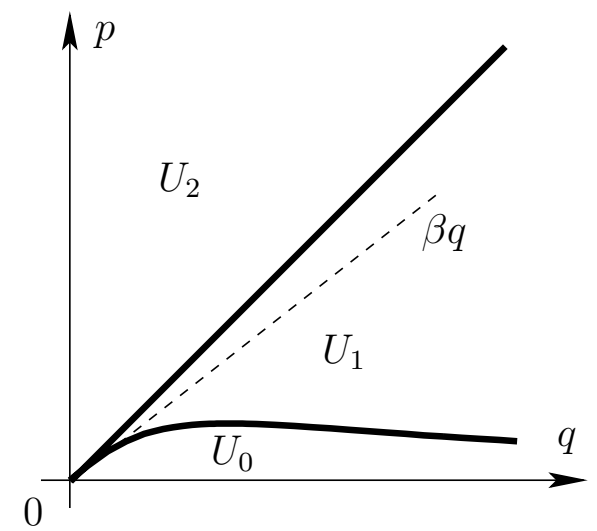

Fig. 11: Typical form of the domains $U_{0}, U_{1}$ and $U_{2}$.

1. if $(p, q) \in U_{0}$, then $\varepsilon \mapsto \eta^{Y}(\varepsilon, T)$ is strictly increasing on $(0,+\infty)$;

2. if $(p, q) \in U_{1}$, then $\varepsilon \mapsto \eta^{Y}(\varepsilon, T)$ has a unique local maximum on $(0,+\infty)$;

3. if $(p, q) \in U_{2}$, then $\varepsilon \mapsto \eta^{Y}(\varepsilon, T)$ has a unique local maximum and vanishes at $\widehat{\varepsilon}=(V-v) / \log \left(\frac{p}{q}\right)$.

Moreover, for $T \rightarrow \infty$ the domain $U_{0} \rightarrow \emptyset$, and the 'optimal tuning' $T_{\eta}=T_{\eta}(\varepsilon)$ which gives the maximum of $\eta^{Y}(\varepsilon, T)$ satisfies

$$
T_{\eta}(\varepsilon)=\frac{\pi}{\sqrt{2 p q}} \sqrt{\frac{v}{V-v}} e^{\frac{V+v}{2 \varepsilon}}\left\{1+\mathcal{O}\left(e^{-\frac{V-v}{\varepsilon}}\right)\right\}
$$

and

$$
\eta^{Y}\left(\varepsilon, T_{\eta}(\varepsilon)\right) \rightarrow \frac{4}{\pi^{2}} \quad \text { as } \quad \varepsilon \rightarrow 0 .
$$

Proof: Statement a) follows directly from (18).

Let us introduce the variable $t=\exp \left(-\frac{V}{\varepsilon}\right) \in[0,1]$. Then $\varphi=p t$ and $\psi=q t^{\beta}$, and

$$
\eta^{Y}(\varepsilon, T)=\eta_{T}^{Y}(t)=\frac{4}{\pi^{2}} \frac{T^{2}\left(p t-q t^{\beta}\right)^{2}}{\left(p t+q t^{\beta}\right)^{2} T^{2}+\pi^{2}}
$$

Taking the derivative of $\eta_{T}^{Y}$ we find that the extrema of $\eta_{T}^{Y}$ are the roots of the equation

$$
t^{\beta}\left(p t-q t^{\beta}\right)\left\{\pi^{2}\left(q \beta-p t^{1-\beta}\right)-2 p q T^{2}(1-\beta) t\left(q t^{\beta}+p t\right)\right\}=0 .
$$

The first parenthesis of $(21)$ gives the root $t=(q / p)^{\frac{1}{1-\beta}}$ which does not depend on $T$, is less than 1 if $p>q$, and corresponds to $\widehat{\varepsilon}=(V-v) / \log \left(\frac{p}{q}\right)$.

Let us show that the function in the second parenthesis of (21) has exactly one root if $(p, q) \in U_{1} \cup U_{2}$, and no root if $(p, q) \in U_{0}$. Denote the second parenthesis of (21) by $F(t)$. Note that $F(0)=\pi^{2} q \beta>0$,

$$
F^{\prime}(t)=-\pi^{2} p(1-\beta) t^{-\beta}-2 p q^{2} T^{2}\left(1-\beta^{2}\right) t^{\beta}-4 p^{2} q T^{2}(1-\beta) t \leq 0 .
$$


Consequently, $F$ is monotonically decreasing on $(0,1]$. Hence, $F$ has a unique root on $t \in(0,1]$ if and only if $F(1) \leq 0$. The latter inequality can be rewritten as the following quadratic inequality w.r.t. $p$

$$
2 p^{2} q T^{2}(1-\beta)+\left(2 q^{2} T^{2}(1-\beta)+\pi^{2}\right) p-q \beta \pi^{2} \geq 0 .
$$

Solving this inequality results in (19). The properties of $\widehat{p}(q)$ follow from a straightforward calculation.

To find an 'optimal tuning' $T=T(\varepsilon)$ we note that $F(t)=0$ is a linear equation in $T^{2}$ from which we get that

$$
T_{\eta}=\frac{\pi}{\sqrt{2 p q}} \sqrt{\frac{\beta}{1-\beta}} t^{-\frac{1+\beta}{2}}\left(\frac{1-\frac{p}{q} \frac{1}{\beta} t^{1-\beta}}{1+\frac{p}{q} t^{1-\beta}}\right)^{\frac{1}{2}} .
$$

It is clear that as $T$ tends to $\infty$, the noise parameter $\varepsilon$ tends to 0 and (23) can be rewritten as

$$
T_{\eta}=\frac{\pi}{\sqrt{2 p q}} \sqrt{\frac{v}{V-v}} e^{\frac{V+v}{2 \varepsilon}}\left\{1+\mathcal{O}\left(e^{-\frac{V-v}{\varepsilon}}\right)\right\} .
$$

Note that for large $T$ the solution exists always, for any $v, V, p, q$, hence $U_{0} \rightarrow \emptyset$ as $\varepsilon \rightarrow 0$. The limit (20) is obtained by inserting (24) into (18).

\section{Stochastic Resonance in Diffusions}

In this section we return to the diffusion in a time-periodic double-well potential. Our aim is to compare the spectral power amplification coefficient of the diffusion with its counterpart for the dynamically adapted continuous-time Markov chain on the twopoint space composed of the metastable states of the diffusion. The invariant density of the diffusion satisfies the forward Kolmogorov (Fokker-Planck) equation, which in this case is a parabolic partial differential equation with antisymmetric boundary conditions. The invariant density is described by a Floquet type expansion along the discrete spectrum of the diffusion's infinitesimal generator. A fine analysis developed in Pavlyukevich [32] built upon the spectral theory of the infinitesimal generator allows to distinguish clearly between the important and the asymptotically vanishing parts in the expansion. The main observation is that due to the double well structure of our potential the first two eigenvalues of the infinitesimal generator associated with the time-invariant potential are zero resp. exponentially small, while the remaining point spectrum is separated from them by a constant positive gap independent of noise intensity. As a consequence, the SPA coefficient which by definition is taken with respect to the invariant measure will receive essential contributions only from the first two terms in the invariant density's expansion. Theorem 4.1 contains the asymptotics of the SPA coefficient, if the noise parameter $\varepsilon$ runs through the intervals $\left[\frac{v+\delta}{\log T}, \frac{2 V}{\log T}\right], \delta>0$. In the large period limit $T \rightarrow \infty$ these intervals shrink in the natural scale on the one hand. Freidlin [17] on the other hand suggests that these are the relevant resonance intervals to look for. This impression is in particular supported by the observation made in Section 3 where we prove that the Markov chain SPA coefficient has a local 
maximum at $\varepsilon \sim \frac{v+V}{2 \log T}$. Surprisingly, it turns out that for the diffusion the SPA tuning curve is either decreasing or increasing on the resonance intervals. This means that the reduction to a Markov chain on the metastable states, however naturally it main retain the dynamical properties of the diffusion, does not preserve optimal tuning effects, at least not for the physicists' favourite measure of quality. The reason for this is hidden in the significance of many small random fluctuations of the diffusion in the potential valley bottoms where it spends most of the time. If we cut off these fluctuations by identifying the valley bottoms with the minima themselves, we obtain a modified SPA coefficient which exactly shows the same resonance effects as the Markov chain in the large period limit (small noise limit).

\subsection{Diffusion with time-periodic drift and its invariant density}

Let us first formulate assumptions concerning the potential function generating our diffusion.

$U(\cdot, \cdot)$ is periodic in time, i.e. $U(\cdot, t)=U(\cdot, t+1)$. We also assume that it is a step-function in time alternating between two spatially antisymmetric states, i.e.

$$
U(x, t)= \begin{cases}U(x), & t \in\left[k, k+\frac{1}{2}\right), \\ U(-x), & t \in\left[k+\frac{1}{2}, k+1\right), \quad k \in \mathbb{Z}_{+} .\end{cases}
$$

The following hypotheses on the geometry of $U$ will be used:

(S) $\quad U \in \mathcal{C}^{\infty}(\mathbb{R})$;

(G) there exists $R>0$ such that $U(x)=x^{4} / 4$ for $|x| \geq R$;

(M) $U$ has exactly two local minima at $x= \pm 1$ and one local maximum at $x=0$; moreover,

$U(-1)=-\frac{V}{2}, \quad U(0)=0, \quad U(1)=-\frac{v}{2}, \quad \frac{2}{3}<\frac{v}{V}<1 ;$

the extrema are non-degenerate, i.e.

$U^{\prime \prime}( \pm 1)=\omega_{ \pm}>0, \quad U^{\prime \prime}(0)=-\omega_{0}<0$.

The condition $\frac{2}{3}<\frac{v}{V}<1$ may seem little transparent at first glance. Note that it only means that the depths of the two potential wells are not too different. The process $X^{\varepsilon, T}$ has continuous trajectories. The fast increase of the potential at infinity guarantees that the process is positively recurrent. This means that for any initial point, the mean time to enter any interval on $\mathbb{R}$ is finite. For details see [27], [18].

In case the process is defined by the SDE (4) there is no invariant measure in the usual sense since the process in not time-homogeneous. On the other hand, the timeperiodicity of the drift $-U^{\prime}(\cdot, t)$ suggests that the law of $X_{t}^{\varepsilon, T}$ should converge to some time-periodic law.

For convenience we rescale time $t \mapsto \frac{t}{2 T}$. To give a rigorous mathematical meaning to this we consider a new two-dimensional process

$$
\mathbf{X}_{t}^{\varepsilon, T}=\left(X_{2 T t}^{\varepsilon, T}, t(\bmod 1)\right), \quad t \geq 0
$$


It takes values on the cylinder $\mathbb{R} \times S^{1}$, and is a time-homogeneous Markov process. This means that it has an invariant measure $\mathbf{m}^{\varepsilon, T}$ on $\mathcal{B}\left(\mathbb{R} \times S^{1}\right)$ with density $\mu^{\varepsilon, T}$, which is the unique positive solution of the forward Kolmogorov equation

$$
A_{\varepsilon, T}^{*} \mu^{\varepsilon, T}(x, \theta)=0, \quad(x, \theta) \in \mathbb{R} \times([0,1),
$$

with the continuity condition

$$
\mu^{\varepsilon, T}(\cdot, 0)=\mu^{\varepsilon, T}(\cdot, 1)
$$

and such that $\int_{0}^{1} \int_{\mathbb{R}} \mu^{\varepsilon, T}(x, \theta) d x d \theta=1$. Note that $\mu^{\varepsilon, T}(\cdot, \theta)$ determines the law of the r.v. $X_{\theta}^{\varepsilon, T}$, and therefore $\int_{\mathbb{R}} \mu^{\varepsilon, T}(x, \theta) d x=1$ for any $\theta \in[0,1]$.

The operator

$$
A_{\varepsilon, T}^{*} f=\frac{\varepsilon}{2} \frac{\partial^{2}}{\partial x^{2}} f+\frac{\partial}{\partial x}\left(\frac{\partial}{\partial x} U f\right)-\frac{1}{2 T} \frac{\partial}{\partial \theta} f, \quad f \in C_{0}^{\infty}\left(\mathbb{R} \times S^{1}\right),
$$

is the formal adjoint of the infinitesimal generator of $\mathbf{X}^{\varepsilon, T}$

$$
A_{\varepsilon, T} f=\frac{\varepsilon}{2} \frac{\partial^{2}}{\partial x^{2}} f-\frac{\partial}{\partial x} U \frac{\partial}{\partial x} f+\frac{1}{2 T} \frac{\partial}{\partial \theta} f, \quad f \in C_{0}^{\infty}\left(\mathbb{R} \times S^{1}\right) .
$$

Taking (25) into account we rewrite (26) and (27) in the form

$$
\left\{\begin{array}{l}
\frac{\varepsilon}{2} \frac{\partial^{2}}{\partial x^{2}} \mu^{\varepsilon, T}+\frac{\partial}{\partial x}\left(\mu^{\varepsilon, T} U^{\prime}\right)=\frac{1}{2 T} \frac{\partial}{\partial \theta} \mu^{\varepsilon, T}, \text { on } \mathbb{R} \times\left(0, \frac{1}{2}\right) \\
\frac{\varepsilon}{2} \frac{\partial^{2}}{\partial x^{2}} \mu^{\varepsilon, T}(x, \theta)+\frac{\partial}{\partial x}\left(\mu^{\varepsilon, T} \bar{U}^{\prime}\right)=\frac{1}{2 T} \frac{\partial}{\partial \theta} \mu^{\varepsilon, T}, \text { on } \mathbb{R} \times\left(\frac{1}{2}, 1\right), \\
\mu^{\varepsilon, T} \text { is positive and continuous on } \mathbb{R} \times[0,1], \\
\int_{\mathbb{R}} \mu^{\varepsilon, T}(x, \theta) d x=1, \quad \theta \in[0,1] .
\end{array}\right.
$$

From the spatial antisymmetry (25) we can immediately deduce a similar antisymmetry property for $\mu^{\varepsilon, T}$.

Proposition 4.1 For $x \in \mathbb{R}$ and $\theta \in\left(0, \frac{1}{2}\right)$ we have

$$
\mu^{\varepsilon, T}(x, \theta)=\mu^{\varepsilon, T}\left(-x, \theta+\frac{1}{2}\right) .
$$

This proposition is analogous to Proposition 3.1 concerning the invariant law of the Markov chain.

Proposition 4.1 together with (28) show that it is enough to find the invariant density on the first half-period, i.e. in the strip $\mathbb{R} \times\left[0, \frac{1}{2}\right]$ where it is a solution of the following boundary-value problem

$$
\left\{\begin{array}{l}
\frac{\varepsilon}{2} \frac{\partial^{2}}{\partial x^{2}} \mu^{\varepsilon, T}+\frac{\partial}{\partial x}\left(\mu^{\varepsilon, T} U^{\prime}\right)=\frac{1}{2 T} \frac{\partial}{\partial \theta} \mu^{\varepsilon, T} \text { on } \mathbb{R} \times\left(0, \frac{1}{2}\right) \\
\mu^{\varepsilon, T}(\cdot, 0)=\mu^{\varepsilon, T}\left(-\cdot, \frac{1}{2}\right) \\
\mu^{\varepsilon, T}>0, \quad \text { on } \mathbb{R} \times\left[0, \frac{1}{2}\right] \\
\int_{\mathbb{R}} \mu^{\varepsilon, T}(x, \theta) d x=1, \quad \theta \in\left[0, \frac{1}{2}\right] .
\end{array}\right.
$$




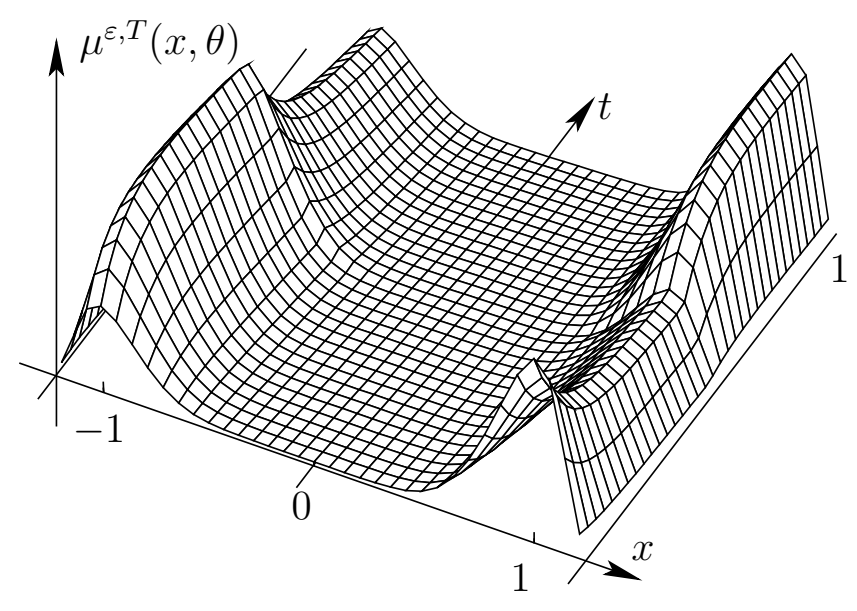

Fig. 12: The invariant density of time-inhomogeneous diffusion $X^{\varepsilon, T}$.

A typical form of the invariant density $\mu^{\varepsilon, T}$ as a function of $x$ and $\theta$ is shown in Fig. 12.

In the following subsection we describe $\mu^{\varepsilon, T}$ in the small noise limit in a Fourier type expansion.

\subsection{Asymptotic expansion of the invariant density}

On the way to solve (29), separation of variables lead us to an eigenvalue problem for the infinitesimal generator of a time-homogeneous diffusion. Assume that a solution $\mu^{\varepsilon, T}$ of the partial differential equation in (29) allows a factorization

$$
\mu^{\varepsilon, T}(x, \theta)=\Psi^{\varepsilon, T}(x) \Theta^{\varepsilon, T}(\theta), \quad x \in \mathbb{R}, \theta \in\left(0, \frac{1}{2}\right) .
$$

Then it follows from (29) that

$$
\frac{\varepsilon}{2} \frac{\left(\Psi^{\varepsilon, T}\right)^{\prime \prime}}{\Psi^{\varepsilon, T}}+U^{\prime} \frac{\left(\Psi^{\varepsilon, T}\right)^{\prime}}{\Psi^{\varepsilon, T}}+U^{\prime \prime}=\frac{1}{2 T} \frac{\dot{\Theta}^{\varepsilon, T}}{\Theta^{\varepsilon, T}}=-\lambda,
$$

where $f^{\prime}=\frac{d f}{d x}$ and $\dot{g}=\frac{d g}{d \theta}$ etc. The constant $\lambda$ does not depend on $x$ and $\theta$ and is an eigenvalue of the differential operator $-L^{*}$, where

$$
L^{*} f=\frac{\varepsilon}{2} f^{\prime \prime}+U^{\prime} f^{\prime}+U^{\prime \prime} f, \quad f \in \mathcal{C}_{0}^{\infty}(\mathbb{R}) .
$$

Due to the geometric properties of $U$ (for details see Pavlyukevich [32]), $L^{*}$ is the formal adjoint of the infinitesimal generator of the time-homogeneous diffusion with potential $U$. As an operator on $\mathcal{L}^{2}(\mathbb{R}, \rho d x)$ with $\rho=e^{\frac{2 U}{\varepsilon}}$ it has a discrete spectrum $\left\{-\lambda_{k}\right\}_{k \geq 0}$ such that $0=\lambda_{0}<\lambda_{1}<\lambda_{2}<\cdots$. Between $\lambda_{1}$ and $\lambda_{2}$ there is a spectral gap. More precisely, there exists $M>0$ and $\varepsilon_{0}>0$ such that

$$
\lambda_{2}(\varepsilon) \geq M, \text { for all } 0<\varepsilon \leq \varepsilon_{0} .
$$


Moreover, (see Pavlyukevich [32] for details), we have $\lambda_{0}=0$, and the first eigenvalue of $L^{*}$ is exponentially small in $\varepsilon$ and equals $\lambda_{1}(\varepsilon)=\frac{\sqrt{\omega_{0} \omega_{+}}}{2 \pi} e^{-\frac{v}{\varepsilon}}(1+\mathcal{O}(\varepsilon))$.

The normalized eigenfunctions $\left\{\frac{\Psi_{k}}{\left\|\Psi_{k}\right\|_{\rho}}\right\}_{k \geq 0}$ provide a complete orthonormal system in $\mathcal{L}^{2}(\mathbb{R}, \rho d x)$ with inner product $(\cdot, \cdot)_{\rho}$ and norm $\|\cdot\|_{\rho}$, i.e.

$$
\int_{\mathbb{R}} \frac{\Psi_{k}(y)}{\left\|\Psi_{k}\right\|_{\rho}} \frac{\Psi_{j}(y)}{\left\|\Psi_{j}\right\|_{\rho}} e^{\frac{2 U(y)}{\varepsilon}} d y=\delta_{k j}, \quad k, j \in \mathbb{Z}_{+}
$$

We know $\Psi_{0}=\rho^{-1}$, up to a multiplicative constant which we choose equal to 1 . We moreover normalize $\Psi_{1}$ so that $\Psi_{1}(1)=e^{\frac{v}{\varepsilon}}$. The fact that $\lambda_{1}(\varepsilon)$ is exponentially small allows us to get rather precise information about the asymptotic properties of the first eigenfunction $\Psi_{1}$ of $L^{*}$ in the small noise limit. In fact, a series expansion of $\Psi_{1}$ in powers of $\lambda_{1}(\varepsilon)$ is used in Pavlyukevich [32] to give precise estimates. Combining these with Laplace's method for the evaluation of singular integrals yields the following estimates which will be important for describing the asymptotic properties of the diffusion's SPA coefficient.

Proposition 4.2 In the small noise limit $\varepsilon \rightarrow 0$ we have

$$
\begin{aligned}
\left\|\Psi_{0}\right\|_{\rho}^{2} & =\sqrt{\frac{\pi \varepsilon}{\omega_{-}}} e^{\frac{V}{\varepsilon}}(1+\mathcal{O}(\varepsilon)), \\
\left\|\Psi_{1}\right\|_{\rho}^{2} & =\sqrt{\frac{\pi \varepsilon}{\omega_{+}}} e^{\frac{v}{\varepsilon}}(1+\mathcal{O}(\varepsilon)), \\
\left\|\bar{\Psi}_{1} e^{2 \frac{\bar{U}-U}{\varepsilon}}\right\|_{\rho}^{2} & =\sqrt{\frac{\pi \varepsilon}{\omega_{-}}} e^{\frac{V}{\varepsilon}}(1+\mathcal{O}(\varepsilon)), \\
\left(\Psi_{1}, \bar{\Psi}_{1}\right)_{\rho} & =-\sqrt{\frac{\pi \varepsilon}{\omega_{+}}} e^{\frac{v}{\varepsilon}}(1+\mathcal{O}(\varepsilon)), \\
\left(\Psi_{1}, \bar{\Psi}_{0}\right)_{\rho} & =\sqrt{\frac{\pi \varepsilon}{\omega_{-}}} e^{\frac{V}{\varepsilon}}(1+\mathcal{O}(\varepsilon)), \\
\int_{\mathbb{R}} y \Psi_{1}(y) d y & =2 \sqrt{\frac{\pi \varepsilon}{\omega_{+}}} e^{\frac{v}{\varepsilon}}(1+\mathcal{O}(\varepsilon)) .
\end{aligned}
$$

Let $\mu^{\varepsilon, T}$ be the unique solution of $(29)$. Consider $\mu^{\varepsilon, T}(\cdot, 0)$ and expand it into the Fourier series with respect to the system $\left\{\Psi_{k}\right\}_{k \geq 0}$

$$
\mu^{\varepsilon, T}(\cdot, 0)=\sum_{k=0}^{\infty} a_{k}^{\varepsilon, T} \frac{\Psi_{k}}{\left\|\Psi_{k}\right\|_{\rho}}
$$

where the Fourier coefficients are determined by the inner products

$$
a_{k}^{\varepsilon, T}=\left(\frac{\Psi_{k}}{\left\|\Psi_{k}\right\|_{\rho}}, \mu^{\varepsilon, T}(\cdot, 0)\right)_{\rho}, \quad k \geq 0 .
$$

Parseval's equality states that

$$
\left\|\mu^{\varepsilon, T}(\cdot, 0)\right\|_{\rho}^{2}=\sum_{k=0}^{\infty}\left(a_{k}^{\varepsilon, T}\right)^{2} .
$$


From the existence and uniqueness theorem for parabolic partial differential equations $[15,33]$ we obtain the following

Proposition $4.3 \mu^{\varepsilon, T}$ can be represented by the series

$$
\mu^{\varepsilon, T}(x, s)=\sum_{k=0}^{\infty} a_{k}^{\varepsilon, T} \frac{\Psi_{k}(x)}{\left\|\Psi_{k}\right\|_{\rho}} \exp \left(-2 T \lambda_{k} s\right), \quad x \in \mathbb{R}, s \in\left[0, \frac{1}{2}\right] .
$$

The coefficients $a_{k}^{\varepsilon, T}$ in the representation (31) can be expressed in terms of the function $\mu^{\varepsilon, T}(\cdot, 0)$. Next we determine the first coefficient explicitly.

Proposition 4.4 For $\varepsilon>0$ and $T>0$

$$
a_{0}^{\varepsilon, T}=a_{0}^{\varepsilon}=\left\|\Psi_{0}\right\|_{\rho}^{-1}=\left(\int_{\mathbb{R}} e^{-\frac{2 U(y)}{\varepsilon}} d y\right)^{-1 / 2} .
$$

Proof: Using the condition that $\int_{\mathbb{R}} \mu^{\varepsilon, T}(x, s) d x=1$ for any $s$ and $\Psi_{0}=e^{-\frac{2 U}{\varepsilon}}$ we find

$$
\begin{aligned}
1 & =\int_{\mathbb{R}} \mu^{\varepsilon, T}(x, 0) e^{\frac{2 U(x)}{\varepsilon}} \frac{\Psi_{0}(x)}{\left\|\Psi_{0}\right\|_{\rho}}\left\|\Psi_{0}\right\|_{\rho} d x=\left\|\Psi_{0}\right\|_{\rho}\left(\mu^{\varepsilon, T}(\cdot, 0), \frac{\Psi_{0}}{\left\|\Psi_{0}\right\|_{\rho}}\right)_{\rho} \\
& =a_{0}^{\varepsilon, T}\left\|\Psi_{0}\right\|_{\rho}=a_{0}^{\varepsilon, T}\left(\int_{\mathbb{R}} e^{-\frac{2 U(y)}{\varepsilon}} d y\right)^{1 / 2} .
\end{aligned}
$$

Corollary 4.1 Let $T>0$ and $s \geq 0$. There exists $\varepsilon_{0}>0$ such that for $0<\varepsilon \leq \varepsilon_{0}$

$$
\left\|\mu^{\varepsilon, T}(\cdot, s)\right\|_{\rho} \geq a_{0}^{\varepsilon} \geq C \varepsilon^{-1 / 4} e^{-\frac{V}{2 \varepsilon}}
$$

Proof: The proof consists in evaluating the integral from (33) by Laplace's method, see $(74)$.

The second coefficient in the expansion (32) cannot be described explicitly. But the following proposition gives an approximation the goodness of which is guaranteed by the spectral gap. Recall that the spatially symmetric counterpart of a function $f: \mathbb{R} \rightarrow \mathbb{R}$ will be denoted by $\bar{f}$, i.e. $\bar{f}(x)=f(-x), x \in \mathbb{R}$.

Proposition 4.5 Let $M$ be the constant marking the spectral gap. There exist $\varepsilon_{0}>0$ such that for $0<\varepsilon \leq \varepsilon_{0}$ and $T>0$ we have

$$
a_{1}^{\varepsilon, T}=\frac{1}{\left\|\Psi_{0}\right\|_{\rho}} \frac{\left(\frac{\bar{\Psi}_{0}}{\left\|\Psi_{0}\right\|_{\rho}}, \frac{\Psi_{1}}{\left\|\Psi_{1}\right\|_{\rho}}\right)_{\rho}+r(\varepsilon, T)}{1-e^{-\lambda_{1} T}\left(\frac{\bar{\Psi}_{1}}{\left\|\Psi_{1}\right\|_{\rho}}, \frac{\Psi_{1}}{\left\|\Psi_{1}\right\|_{\rho}}\right)_{\rho}},
$$

where

$$
|r(\varepsilon, T)| \leq e^{-M T}\left\|\mu^{\varepsilon, T}(\cdot, 0)\right\|_{\rho} \cdot\left\|\frac{\bar{\Psi}_{1}}{\left\|\Psi_{1}\right\|_{\rho}} e^{\frac{2(\bar{U}-U)}{\varepsilon}}\right\|_{\rho} .
$$


Proof: From (32) and the boundary condition $\mu^{\varepsilon, T}(x, 0)=\mu^{\varepsilon, T}\left(-x, \frac{1}{2}\right), x \in \mathbb{R}$, we obtain

$$
\sum_{k=0}^{\infty} a_{k}^{\varepsilon, T} \frac{\Psi_{k}}{\left\|\Psi_{k}\right\|_{\rho}}=\sum_{k=0}^{\infty} a_{k}^{\varepsilon, T} e^{-\lambda_{k} T} \frac{\bar{\Psi}_{k}}{\left\|\Psi_{k}\right\|_{\rho}}
$$

Multiplying both sides of (36) by $e^{\frac{2 U}{\varepsilon}} \frac{\Psi_{1}}{\left\|\Psi_{1}\right\|_{\rho}}$, integrating, and using (30) gives

$$
a_{1}^{\varepsilon, T}=a_{0}^{\varepsilon}\left(\frac{\bar{\Psi}_{0}}{\left\|\Psi_{0}\right\|_{\rho}}, \frac{\Psi_{1}}{\left\|\Psi_{1}\right\|_{\rho}}\right)_{\rho}+a_{1}^{\varepsilon, T} e^{-\lambda_{1} T}\left(\frac{\bar{\Psi}_{1}}{\left\|\Psi_{1}\right\|_{\rho}}, \frac{\Psi_{1}}{\left\|\Psi_{1}\right\|_{\rho}}\right)_{\rho}+r(\varepsilon, T),
$$

where

$$
r(\varepsilon, T)=\sum_{k=2}^{\infty} a_{k}^{\varepsilon, T} e^{-\lambda_{k} T}\left(\frac{\bar{\Psi}_{k}}{\left\|\Psi_{k}\right\|_{\rho}}, \frac{\Psi_{1}}{\left\|\Psi_{1}\right\|_{\rho}}\right)_{\rho} .
$$

This implies (34). To estimate the remainder term $r$, we now use the spectral gap result. We may choose $\varepsilon_{0}$ such that for $\varepsilon \leq \varepsilon_{0}$ the third eigenvalue $\lambda_{2} \geq M>0$. Hence

$$
\begin{aligned}
|r(\varepsilon, T)| & \leq e^{-M T} \sum_{k=2}^{\infty}\left|a_{k}^{\varepsilon, T}\right|\left|\left(\frac{\bar{\Psi}_{k}}{\left\|\Psi_{k}\right\|_{\rho}}, \frac{\Psi_{1}}{\left\|\Psi_{1}\right\|_{\rho}}\right)_{\rho}\right| \\
& \leq e^{-M T}\left[\sum_{k=2}^{\infty}\left(a_{k}^{\varepsilon, T}\right)^{2}\right]^{1 / 2}\left[\sum_{k=2}^{\infty}\left(\frac{\bar{\Psi}_{k}}{\left\|\Psi_{k}\right\|_{\rho}}, \frac{\Psi_{1}}{\left\|\Psi_{1}\right\|_{\rho}}\right)_{\rho}^{2}\right]^{1 / 2} .
\end{aligned}
$$

The inner products can be rewritten in the following form:

$$
\begin{aligned}
\left(\frac{\bar{\Psi}_{k}}{\left\|\Psi_{k}\right\|_{\rho}}, \frac{\Psi_{1}}{\left\|\Psi_{1}\right\|_{\rho}}\right)_{\rho} & =\int_{\mathbb{R}} \frac{\bar{\Psi}_{k}}{\left\|\Psi_{k}\right\|_{\rho}} \frac{\Psi_{1}}{\left\|\Psi_{1}\right\|_{\rho}} e^{\frac{2 U}{\varepsilon}} d x \\
& =\int_{\mathbb{R}} \frac{\Psi_{k}}{\left\|\Psi_{k}\right\|_{\rho}} \frac{\bar{\Psi}_{1}}{\left\|\Psi_{1}\right\|_{\rho}} e^{\frac{2 \bar{U}}{\varepsilon}} e^{-\frac{2 U}{\varepsilon}} e^{\frac{2 U}{\varepsilon}} d x \\
& =\left(\frac{\bar{\Psi}_{1}}{\left\|\Psi_{1}\right\|_{\rho}} e^{\frac{2(\bar{U}-U)}{\varepsilon}}, \frac{\Psi_{k}}{\left\|\Psi_{k}\right\|_{\rho}}\right)_{\rho}
\end{aligned}
$$

Two applications of Parseval's equality complete the estimate (35).

The only function not even approximately known which appears in the estimate $(35)$ is $\mu^{\varepsilon, T}(\cdot, 0)$. The following proposition provides an upper bound for its $\rho$-norm in terms of the explicit function $\bar{\rho}^{-1}=e^{-\frac{2 \bar{U}}{\varepsilon}}$. Denote $c=\int_{\mathbb{R}} \rho^{-1}(y) d y=\left(a_{0}^{\varepsilon}\right)^{-2}$. Then, the function $\bar{\rho}^{-1} / c$ is the invariant density of the time-homogeneous diffusion in the potential $\bar{U}(x)=U(-x), x \in \mathbb{R}$. The spectral gap between $\lambda_{0}=0$ and $\lambda_{1}$ of the corresponding infinitesimal generator implies that the law of the diffusion converges to the invariant law exponentially fast and determines the rate of convergence. This indicates that the density $\mu^{\varepsilon, T}(\cdot, 0)=\mu^{\varepsilon, T}(\cdot, 1)$ should be close to $\bar{\rho}^{-1} / c$ if $T$ is large enough. One can obtain the following estimate for the norm.

Proposition 4.6 For any $\delta>0$ there exist $T_{0}, \varepsilon_{0}>0$ such that for $T \geq T_{0}, \varepsilon \in$ $\left[\frac{v+\delta}{\log T}, \varepsilon_{0}\right]$

$$
\left\|\mu^{\varepsilon, T}(\cdot, 0)\right\|_{\rho} \leq 6\left\|\bar{\rho}^{-1} / c\right\|_{\rho} .
$$


Proof: Combining the triangle inequality with the inequality $(a+b)^{2} \leq 2\left(a^{2}+b^{2}\right)$, $a, b \in \mathbb{R}$, gives

$$
\left\|\mu^{\varepsilon, T}(\cdot, 0)\right\|_{\rho}^{2} \leq 2\left\|\mu^{\varepsilon, T}(\cdot, 0)-\frac{\bar{\rho}^{-1}}{c}\right\|_{\rho}^{2}+2\left\|\frac{\bar{\rho}^{-1}}{c}\right\|_{\rho}^{2} .
$$

For $n \in \mathbb{N}$, denote by $P_{n}^{\Psi}$ the orthogonal projector on the orthogonal complement of the span of the first $n$ eigenfunctions $\Psi_{0}, \ldots, \Psi_{n-1}$. Let us estimate the first summand in (38). Using the boundary condition in (29) we obtain

$$
\begin{aligned}
\| \mu^{\varepsilon, T} & (\cdot, 0)-\frac{\bar{\rho}^{-1}}{c}\left\|_{\rho}^{2}=\right\| \bar{\mu}^{\varepsilon, T}\left(\cdot, \frac{1}{2}\right)-\frac{\bar{\rho}^{-1}}{c}\left\|_{\rho}^{2}=\right\| \mu^{\varepsilon, T}\left(\cdot, \frac{1}{2}\right)-\frac{\rho^{-1}}{c} \|_{\bar{\rho}}^{2} \\
& =\int_{\mathbb{R}}\left(P_{1}^{\Psi} \mu^{\varepsilon, T}\left(\cdot, \frac{1}{2}\right)\right)^{2} \bar{\rho} d y=\int_{\mathbb{R}}\left|P_{1}^{\Psi} \mu^{\varepsilon, T}\left(\cdot, \frac{1}{2}\right)\right| \cdot\left|\mu^{\varepsilon, T}\left(\cdot, \frac{1}{2}\right)-\frac{\rho^{-1}}{c}\right| \bar{\rho} d y \\
& \leq \int_{\mathbb{R}}\left|P_{1}^{\Psi} \mu^{\varepsilon, T}\left(\cdot, \frac{1}{2}\right)\right| \mu^{\varepsilon, T}\left(\cdot, \frac{1}{2}\right) \bar{\rho} d y+\int_{\mathbb{R}}\left|P_{1}^{\Psi} \mu^{\varepsilon, T}\left(\cdot, \frac{1}{2}\right)\right| \frac{\rho^{-1}}{c} \bar{\rho} d y \\
& \leq \int_{\mathbb{R}}\left|P_{1}^{\Psi} \mu^{\varepsilon, T}\left(\cdot, \frac{1}{2}\right)\right| \mu^{\varepsilon, T}\left(\cdot, \frac{1}{2}\right) \bar{\rho} \rho^{-1} \rho d y+\int_{\mathbb{R}}\left|P_{1}^{\Psi} \mu^{\varepsilon, T}\left(\cdot, \frac{1}{2}\right)\right| \frac{\rho^{-2}}{c} \bar{\rho} \rho d y \\
& \leq\left\|P_{1}^{\Psi} \mu^{\varepsilon, T}\left(\cdot, \frac{1}{2}\right)\right\|_{\rho}\left[\int_{\mathbb{R}}\left(\mu^{\varepsilon, T}\left(\cdot, \frac{1}{2}\right)\right)^{2}\left(\bar{\rho} \rho^{-1}\right)^{2} \rho d y\right]^{\frac{1}{2}}+ \\
& +\left\|P_{1}^{\Psi} \mu^{\varepsilon, T}\left(\cdot, \frac{1}{2}\right)\right\|_{\rho}\left[\int_{\mathbb{R}}\left(\frac{\bar{\rho} \rho^{-2}}{c}\right)^{2} \rho d y\right]^{\frac{1}{2}} \\
& \leq e^{-\lambda_{1} T} \max \left\{\bar{\rho} \rho^{-1}\right\} \cdot\left\|\mu^{\varepsilon, T}(\cdot, 0)\right\|_{\rho}^{2}+\frac{e^{-\lambda_{1} T}}{c}\left[\int_{\mathbb{R}} \bar{\rho}^{2} \rho^{-3} d y\right]^{\frac{1}{2}}\left\|\mu^{\varepsilon, T}(\cdot, 0)\right\|_{\rho} .
\end{aligned}
$$

Taking into account the latter inequality and (38) we obtain a quadratic inequality for $\left\|\mu^{\varepsilon, T}(\cdot, 0)\right\|_{\rho}$

$$
\begin{aligned}
& \left(1-2 e^{-\lambda_{1} T} \max \left\{\bar{\rho} \rho^{-1}\right\}\right)\left\|\mu^{\varepsilon, T}(\cdot, 0)\right\|_{\rho}^{2} \\
& -2 \frac{e^{-\lambda_{1} T}}{c}\left[\int_{\mathbb{R}} \bar{\rho}^{2} \rho^{-3} d y\right]^{\frac{1}{2}}\left\|\mu^{\varepsilon, T}(\cdot, 0)\right\|_{\rho}-2\left\|\frac{\bar{\rho}^{-1}}{c}\right\|_{\rho}^{2} \leq 0 .
\end{aligned}
$$

Let us estimate the coefficients of (39) and thus find an upper bound for $\left\|\mu^{\varepsilon, T}(\cdot, 0)\right\|_{\rho}$.

Let $\varepsilon_{0}$ be given by the spectral gap condition. For any $\delta>0$ and $T$ large enough to be specified later let $\frac{v+\delta}{\log T} \leq \varepsilon \leq \varepsilon_{0}$. Then for some $C>0$, recalling the asymptotics of $\lambda_{1}(\varepsilon)$

$$
e^{-\lambda_{1} T} \leq \exp \left\{-\frac{\sqrt{\omega_{0} \omega_{+}}}{2 \pi} T^{-\frac{v}{v+\delta}} T\left(1+\mathcal{O}\left(\frac{1}{\log T}\right)\right)\right\} \leq \exp \left\{-C T^{\frac{\delta}{v+\delta}}\right\} .
$$

This expression tends to 0 exponentially fast as $T \rightarrow \infty$. Moreover,

$$
\max \left\{\bar{\rho} \rho^{-1}\right\}=\exp \left\{\frac{2}{\varepsilon} \max (U(-x)-U(x))\right\}=\exp \left\{\frac{\alpha}{\varepsilon}\right\},
$$

where $\alpha=2 \max \{U(-x)-U(x)\} \geq V-v>0$ is a finite number, given by the potential $U$. For $\varepsilon \geq \frac{v+\delta}{\log T}$ we obtain

$$
\max \left\{\bar{\rho} \rho^{-1}\right\} \leq T^{\frac{\alpha}{v+\delta}}
$$


Similarly, let $\beta^{\prime}=2 \max \{2 U(-x)-3 U(x)\} \geq 3 V-2 v>0$. Then, using Laplace's method, see (74), and recalling $(\mathbf{G})$ we can estimate for some $C_{1}>0$

$$
\frac{1}{c}\left[\int_{\mathbb{R}} \bar{\rho}^{2} \rho^{-3} d y\right]^{\frac{1}{2}} \leq C_{1} \sqrt{\varepsilon} e^{\frac{\beta^{\prime}-V}{\varepsilon}} \leq T^{\frac{\beta}{v+\delta}}
$$

for $T$ large enough, $\varepsilon \geq \frac{v+\delta}{\log T}$ and some $\beta>\beta^{\prime}-V \geq 2(V-v)>0$.

Since $\max \{U(x)-2 U(-x)\} \geq V-\frac{v}{2}>0$, the free term of (39) is estimated for $\frac{v+\delta}{\log T} \leq \varepsilon \leq \varepsilon_{0}$ by

$$
\left\|\frac{\bar{\rho}^{-1}}{c}\right\|_{\rho}^{2}=\frac{1}{c^{2}} \int_{\mathbb{R}} e^{\frac{2}{\varepsilon}(U(y)-2 U(-y))} d y \geq C_{2} \log T T^{-\frac{v}{v+\delta}},
$$

where $C_{2}$ is a positive constant. This estimate means that the norm $\left\|\bar{\rho}^{-1} / c\right\|_{\rho}$ decays in $T$ not faster than polynomially.

Using (38), (40), (41) and (42) implies that (39) holds if the following inequality holds:

$$
\left\|\mu^{\varepsilon, T}(\cdot, 0)\right\|_{\rho}^{2}\left(1-2 e^{-C T^{\frac{\delta}{v+\delta}}} T^{\frac{\alpha}{v+\delta}}\right)-2 e^{-C T^{\frac{\delta}{v+\delta}}} T^{\frac{\beta}{v+\delta}}\left\|\mu^{\varepsilon, T}(\cdot, 0)\right\|_{\rho}-2\left\|\frac{\bar{\rho}^{-1}}{c}\right\|_{\rho}^{2} \leq 0 .
$$

Consequently,

$$
\begin{aligned}
\left\|\mu^{\varepsilon, T}(\cdot, 0)\right\|_{\rho} & \leq \frac{e^{-C T^{\frac{\delta}{v+\delta}}} T^{\frac{\beta}{v+\delta}}+\sqrt{\left(e^{-C T^{\frac{\delta}{v+\delta}}} T^{\frac{\beta}{v+\delta}}\right)^{2}+2\left\|\frac{\bar{\rho}^{-1}}{c}\right\|_{\rho}^{2}\left(1-2 e^{-C T^{\frac{\delta}{v+\delta}}} T^{\frac{\alpha}{v+\delta}}\right)}}{1-2 e^{-C T^{\frac{\delta}{v+\delta}}} T^{\frac{\alpha}{v+\delta}}} \\
& \leq 2\left(\left\|\frac{\bar{\rho}^{-1}}{c}\right\|_{\rho}+\sqrt{\left\|\frac{\bar{\rho}^{-1}}{c}\right\|_{\rho}^{2}+2\left\|\frac{\bar{\rho}^{-1}}{c}\right\|_{\rho}^{2}}\right) \\
& \leq 6\left\|\frac{\bar{\rho}^{-1}}{c}\right\|_{\rho}
\end{aligned}
$$

for $T \geq T_{0}$, where $T_{0}$ is the minimal value for which $\frac{v+\delta}{\log T} \leq \varepsilon_{0}$,

$$
1-2 e^{-C T^{\frac{\delta}{v+\delta}}} T^{\frac{\alpha}{v+\delta}} \geq \frac{1}{2}
$$

and

$$
e^{-C T^{\frac{\delta}{v+\delta}}} T^{\frac{\beta}{v+\delta}} \leq C_{1} \log T T^{-\frac{v}{v+\delta}} \leq\left\|\frac{\bar{\rho}^{-1}}{c}\right\|_{\rho}
$$

\subsection{Spectral power amplification}

This and the following subsections are devoted to the problem of stochastic resonance for the diffusion (4). As a measure of quality of tuning, we shall consider the spectral power amplification coefficient, just as for the two-state reduction in Section 3. Of 
course, to make the two-state chain a consistent model of the reduced diffusion dynamics, we now have to adapt the pre-factors $p$ and $q$ to recover the true asymptotics of Kramers' times hidden in the precise description of $\lambda_{1}$. In this setting, our original plan was to prove that the resonance point obtained in Section 3 for the SPA coefficient of the Markov chain determines and thus characterizes a resonance point for the diffusion, in the small noise limit. To our surprise, this turns out not to be the case, as we shall now make precise.

The spectral power amplification coefficient is defined by

$$
\eta^{X}(\varepsilon, T)=\left|\int_{0}^{1} \mathbf{E}_{\mu} X_{2 T s}^{\varepsilon, T} e^{2 \pi i s} d s\right|^{2} .
$$

It will be compared with the analogous coefficient (17) of the Markov chain. Hereby we take the average with respect to the invariant law of the diffusion the density of which is $\mu^{\varepsilon, T}$.

The SPA coefficient describes the energy of the averaged trajectory carried by the spectral component of period $2 T$, i.e. the period of the 'input signal'.

First, let us rewrite and simplify (44). We get

$$
\begin{aligned}
\eta^{X} & (\varepsilon, T)=\left|\int_{0}^{1} \mathbf{E}_{\mu} X_{2 T s}^{\varepsilon, T} e^{2 \pi i s} d s\right|^{2} \\
& =\left|\int_{0}^{1 / 2} e^{2 \pi i s} \int_{\mathbb{R}} x \mu^{\varepsilon, T}(x, s) d x d s+\int_{1 / 2}^{1} e^{2 \pi i s} \int_{\mathbb{R}} x \mu^{\varepsilon, T}(x, s) d x d s\right|^{2} \\
& =\left|\int_{0}^{1 / 2} e^{2 \pi i s} \int_{\mathbb{R}} x \mu^{\varepsilon, T}(x, s) d x d s+e^{\pi i} \int_{0}^{1 / 2} e^{2 \pi i s} \int_{\mathbb{R}} x \mu^{\varepsilon, T}\left(x, s+\frac{1}{2}\right) d x d s\right|^{2} \\
& =\left|\int_{0}^{1 / 2} e^{2 \pi i s} \int_{\mathbb{R}} x \mu^{\varepsilon, T}(x, s) d x d s-\int_{0}^{1 / 2} e^{2 \pi i s} \int_{\mathbb{R}} x \mu^{\varepsilon, T}(-x, s) d x d s\right|^{2} \\
& =4\left|\int_{0}^{1 / 2} e^{2 \pi i s} \int_{\mathbb{R}} x \mu^{\varepsilon, T}(x, s) d x d s\right|^{2}=4\left|S^{X}(\varepsilon, T)\right|^{2} .
\end{aligned}
$$

Using (32) we find

$$
\begin{aligned}
S^{X}(\varepsilon, T)= & \int_{0}^{1 / 2} e^{2 \pi i s} \int_{\mathbb{R}} x \mu^{\varepsilon, T}(x, s) d x d s= \\
& a_{0}^{\varepsilon} \int_{0}^{1 / 2} e^{2 \pi i s} d s \int_{\mathbb{R}} x \frac{\Psi_{0}(x)}{\left\|\Psi_{0}\right\|_{\rho}} d x+ \\
& a_{1}^{\varepsilon, T} \int_{0}^{1 / 2} e^{2 \pi i s} e^{-2 \lambda_{1} T s} d s \int_{\mathbb{R}} x \frac{\Psi_{1}(x)}{\left\|\Psi_{1}\right\|_{\rho}} d x+r_{1}(\varepsilon, T),
\end{aligned}
$$

where

$$
r_{1}(\varepsilon, T)=\int_{0}^{1 / 2} e^{2 \pi i s} \int_{\mathbb{R}} x \sum_{k=2}^{\infty} a_{k}^{\varepsilon, T} \frac{\Psi_{k}(x)}{\left\|\Psi_{k}\right\|_{\rho}} \exp \left(-2 T \lambda_{k} s\right) d x d s
$$


In the sequel, we shall occasionally use the symbol $x$ to also denote the identity function on $\mathbb{R}, x \mapsto x$.

Proposition 4.7 There exists $\varepsilon_{0}>0$ such that for $0<\varepsilon \leq \varepsilon_{0}$ and $T>0$ we have

$$
\left|r_{1}(\varepsilon, T)\right| \leq \frac{1}{2 T M}\left\|\mu^{\varepsilon, T}(\cdot, 0)\right\|_{\rho} \cdot\left\|x e^{-\frac{2 U}{\varepsilon}}\right\|_{\rho},
$$

with $M$ from the spectral gap condition.

Proof: Indeed, employing the spectral gap for the fourth inequality, we deduce

$$
\begin{aligned}
\left|r_{1}(\varepsilon, T)\right| & \leq \sum_{k=2}^{\infty}\left|a_{k}^{\varepsilon, T}\right| \int_{0}^{1 / 2} e^{-2 T \lambda_{k} s} d s\left|\int_{\mathbb{R}} x \frac{\Psi_{k}(x)}{\left\|\Psi_{k}\right\|_{\rho}} d x\right| \\
& \leq \sum_{k=2}^{\infty}\left|a_{k}^{\varepsilon, T}\right|\left|\int_{\mathbb{R}} x \frac{\Psi_{k}(x)}{\left\|\Psi_{k}\right\|_{\rho}} d x\right| \frac{1-\exp \left(-\lambda_{k} T\right)}{2 \lambda_{k} T} \\
& \leq \frac{1}{2 \lambda_{2} T} \sum_{k=2}^{\infty}\left|a_{k}^{\varepsilon, T}\right|\left|\int_{\mathbb{R}} x \frac{\Psi_{k}(x)}{\left\|\Psi_{k}\right\|_{\rho}} d x\right| \\
& \leq \frac{1}{2 M T}\left[\sum_{k=2}^{\infty}\left(a_{k}^{\varepsilon, T}\right)^{2} \sum_{k=2}^{\infty}\left(x e^{-\frac{2 U}{\varepsilon}}, \frac{\Psi_{k}}{\left\|\Psi_{k}\right\|_{\rho}}\right)_{\rho}^{2}\right]^{1 / 2}
\end{aligned}
$$

It remains to apply Parseval's equality.

We next determine the leading term of $S^{X}(\varepsilon, T)$. We use formula (45), and recall that the coefficients $a_{0}^{\varepsilon}$ and $a_{1}^{\varepsilon, T}$ are obtained in Propositions 4.4 resp. 4.5 .

Lemma 4.1 For any $\delta>0$ there exist $T_{0}, \varepsilon_{0}>0$ such that for $T \geq T_{0}$ and $\varepsilon \in\left[\frac{v+\delta}{\log T}, \varepsilon_{0}\right]$

$$
\begin{aligned}
& S^{X}(\varepsilon, T)=\frac{i}{\pi} \frac{\int_{\mathbb{R}} y \Psi_{0}(y) d y}{\left\|\Psi_{0}\right\|_{\rho}^{2}} \\
& \quad-\frac{1+e^{-\lambda_{1} T}}{2\left(\pi i-\lambda_{1} T\right)} \frac{\int_{\mathbb{R}} y \Psi_{1}(y) d y}{\left\|\Psi_{0}\right\|_{\rho}^{2}} \frac{\left(\bar{\Psi}_{0}, \Psi_{1}\right)_{\rho}}{\left\|\Psi_{1}\right\|_{\rho}^{2}-e^{-\lambda_{1} T}\left(\bar{\Psi}_{1}, \Psi_{1}\right)_{\rho}}+r_{2}(\varepsilon, T),
\end{aligned}
$$

where

$$
\left|r_{2}(\varepsilon, T)\right| \leq \frac{6}{M T}\left(\int_{\mathbb{R}} e^{-\frac{2 U(y)}{\varepsilon}} d y\right)^{-1}\left\|e^{-\frac{2 \bar{U}}{\varepsilon}}\right\|_{\rho} \cdot\left\|x e^{-\frac{2 U}{\varepsilon}}\right\|_{\rho}
$$

and $M$ is the spectral gap constant.

Proof: The leading term of (48) is obtained from (45) by integration in $s \in\left[0, \frac{1}{2}\right]$. Let us estimate the error term $r_{2}(\varepsilon, T)$ which is composed of $r_{1}(\varepsilon, T)$ and $r(\varepsilon, T)$ from 
formula (34). Using Proposition 4.7 and Proposition 4.5, we get

$$
\begin{aligned}
\left|r_{2}(\varepsilon, T)\right| & \leq\left|r_{1}(\varepsilon, T)\right| \\
& +\frac{|r(\varepsilon, T)|}{\left\|\Psi_{0}\right\|_{\rho}\left|1-e^{-\lambda_{1} T}\left(\frac{\bar{\Psi}_{1}}{\left\|\Psi_{1}\right\|_{\rho}}, \frac{\Psi_{1}}{\left\|\Psi_{1}\right\|_{\rho}}\right)_{\rho}\right|}\left|\frac{1+e^{-\lambda_{1} T}}{2\left(\pi i-\lambda_{1} T\right)} \int_{\mathbb{R}} y \frac{\Psi_{1}(y)}{\left\|\Psi_{1}\right\|_{\rho}} d y\right| \\
& \leq \frac{1}{2 M T}\left\|\mu^{\varepsilon, T}(\cdot, 0)\right\|_{\rho} \cdot\left\|x e^{-\frac{2 U}{\varepsilon}}\right\|_{\rho} \\
& +\frac{e^{-M T}}{\left\|\Psi_{0}\right\|_{\rho}} \frac{\left\|\mu^{\varepsilon, T}(\cdot, 0)\right\|_{\rho} \cdot\left\|\bar{\Psi}_{1} e^{2 \frac{\bar{U}-U}{\varepsilon}}\right\|_{\rho}}{\left\|\Psi_{1}\right\|_{\rho}^{2}-e^{-\lambda_{1} T}\left(\bar{\Psi}_{1}, \Psi_{1}\right)_{\rho} \mid}\left|\int_{\mathbb{R}} y \Psi_{1}(y) d y\right| \\
& =\frac{1}{2 M T}\left\|\mu^{\varepsilon, T}(\cdot, 0)\right\|_{\rho} \\
& \times\left(\left\|x e^{-\frac{2 U}{\varepsilon}}\right\|_{\rho}+\frac{2 M T e^{-M T}}{\left\|\Psi_{0}\right\|_{\rho}} \frac{\left\|\bar{\Psi}_{1} e^{2 \frac{\bar{U}-U}{\varepsilon}}\right\|_{\rho} \cdot\left|\int_{\mathbb{R}} y \Psi_{1}(y) d y\right|}{\left|\left\|\Psi_{1}\right\|_{\rho}^{2}-e^{-\lambda_{1} T}\left(\bar{\Psi}_{1}, \Psi_{1}\right)_{\rho}\right|}\right) .
\end{aligned}
$$

Note that for $T$ large enough Laplace's method yields

$$
C_{1} \leq \max _{\varepsilon \in\left[\frac{v+\delta}{\log T}, \varepsilon_{0}\right]}\left\|x e^{-\frac{2 U}{\varepsilon}}\right\|_{\rho}=\max _{\varepsilon \in\left[\frac{v+\delta}{\log T}, \varepsilon_{0}\right]}\left(\int_{\mathbb{R}} y^{2} e^{-\frac{2 U(y)}{\varepsilon}} d y\right)^{\frac{1}{2}} \leq C_{2} T^{\frac{V}{2(v+\delta)}}
$$

for some positive constants $C_{1}$ and $C_{2}$. This means that $\left\|x e^{-\frac{2 U}{\varepsilon}}\right\|_{\rho}$ is bounded on $\left[\frac{v+\delta}{\log T}, \varepsilon_{0}\right]$ polynomially in $T$. Next we show that due to the factor $e^{-M T}$ the second summand in the parenthesis in (50) is exponentially small in $T$.

Using Proposition 4.2 we estimate in $\varepsilon \in\left[\frac{v+\delta}{\log T}, \varepsilon_{0}\right]$ for $T$ large enough and with universal constant $C$ eventually changing from line to line

$$
\begin{aligned}
\frac{2 M T e^{-M T}}{\left\|\Psi_{0}\right\|_{\rho}} & \frac{\left\|\bar{\Psi}_{1} e^{2 \frac{\bar{U}-U}{\varepsilon}}\right\|_{\rho} \cdot\left|\int_{\mathbb{R}} y \Psi_{1}(y) d y\right|}{\left|\left\|\Psi_{1}\right\|_{\rho}^{2}-e^{-\lambda_{1} T}\left(\bar{\Psi}_{1}, \Psi_{1}\right)_{\rho}\right|} \\
& \leq C T e^{-M T} \frac{\sqrt[4]{\varepsilon} e^{\frac{V}{2 \varepsilon}} \cdot \sqrt{\varepsilon} e^{\frac{v}{\varepsilon}}}{\sqrt[4]{\varepsilon} e^{\frac{V}{2 \varepsilon}} \sqrt{\varepsilon} e^{\frac{v}{\varepsilon}}\left(1+e^{-\lambda_{1} T}\right)} \\
& \leq C T e^{-M T} \leq\left\|x e^{-\frac{2 U}{\varepsilon}}\right\|_{\rho} .
\end{aligned}
$$

Applying the previous formula and the inequality $\left\|\mu^{\varepsilon, T}(\cdot, 0)\right\|_{\rho} \leq 6\left\|\bar{\rho}^{-1} / c\right\|_{\rho}$ obtained in Proposition 4.6 to (50) completes the proof.

Next we determine the value of the spectral power amplification coefficient $\eta^{X}(\varepsilon, T)$ in the interval $\left[\frac{v+\delta}{\log T}, \varepsilon_{0}\right]$ for $\delta>0$ and large $T$. For abbreviation of the leading term, let us set

$$
\begin{aligned}
& b_{0}=b_{0}^{\varepsilon}=\frac{\int_{\mathbb{R}} y \Psi_{0}(y) d y}{\left\|\Psi_{0}\right\|_{\rho}^{2}}=\frac{\int_{\mathbb{R}} y e^{-\frac{2 U(y)}{\varepsilon}} d y}{\int_{\mathbb{R}} e^{-\frac{2 U(y)}{\varepsilon}} d y} \\
& b_{1}=b_{1}^{\varepsilon, T}=-\frac{1+e^{-\lambda_{1} T}}{2} \frac{\int_{\mathbb{R}} y \Psi_{1}(y) d y}{\left\|\Psi_{0}\right\|_{\rho}^{2}} \frac{\left(\bar{\Psi}_{0}, \Psi_{1}\right)_{\rho}}{\left\|\Psi_{1}\right\|_{\rho}^{2}-e^{-\lambda_{1} T}\left(\bar{\Psi}_{1}, \Psi_{1}\right)_{\rho}} .
\end{aligned}
$$


Then (48) can be rewritten in the form

$$
S^{X}(\varepsilon, T)=\frac{i}{\pi} b_{0}+\frac{1}{\pi i-\lambda_{1} T} b_{1}+r_{2}(\varepsilon, T)
$$

and therefore

$$
\eta^{X}(\varepsilon, T)=4\left|S^{X}(\varepsilon, T)\right|^{2}=\frac{4}{\pi^{2}} \frac{b_{0}^{2}\left(\lambda_{1} T\right)^{2}}{\pi^{2}+\left(\lambda_{1} T\right)^{2}}+4 \frac{\left(b_{1}-b_{0}\right)^{2}}{\pi^{2}+\left(\lambda_{1} T\right)^{2}}+r_{3}(\varepsilon, T),
$$

where

$$
r_{3}=4\left|r_{2}\right|^{2}+8 \operatorname{Re}\left(s^{X} r_{2}^{*}\right),
$$

'*' denotes the complex conjugate and $s^{X}=\frac{i}{\pi} b_{0}+\frac{1}{\pi i-\lambda_{1} T} b_{1}$ is the leading term of $S^{X}$. We see that $\eta^{X}$ is represented as a sum of three terms. Let us recall its Markov chain counterpart $\eta^{Y}$ determined in Proposition 3.3. We have

$$
\eta^{Y}(\varepsilon, T)=\frac{4}{\pi^{2}} \frac{T^{2}(\varphi-\psi)^{2}}{\pi^{2}+(\varphi+\psi)^{2} T^{2}}
$$

It is clearly seen, that the first leading term of (52) is similar to (54). The correspondence were exact if $\lambda_{1} \approx \psi \pm \varphi$ and $b_{0} \approx 1$.

In the following Lemma we obtain asymptotic estimates for $b_{0}$ and $b_{1}$.

Lemma 4.2 There is $\varepsilon_{0}>0$ such that for $\varepsilon \leq \varepsilon_{0}$ we have

$$
\begin{aligned}
& b_{0}=-1-\frac{U^{(3)}(-1)}{4 \omega_{-}^{2}} \varepsilon+\mathcal{O}\left(\varepsilon^{2}\right), \\
& b_{1}=-1+\mathcal{O}(\varepsilon),
\end{aligned}
$$

and consequently

$$
\begin{aligned}
& b_{0}^{2}=1+\frac{U^{(3)}(-1)}{2 \omega_{-}^{2}} \varepsilon+\mathcal{O}\left(\varepsilon^{2}\right), \\
& \left(b_{1}-b_{0}\right)^{2}=\mathcal{O}\left(\varepsilon^{2}\right)
\end{aligned}
$$

Proof: We use Laplace's method to obtain the asymptotic expansions for the integrals $\int_{\mathbb{R}} e^{-\frac{2 U(y)}{\varepsilon}} d y$ and $\int_{\mathbb{R}} y e^{-\frac{2 U(y)}{\varepsilon}} d y$; compare the formulae $(73)$ and (75) in the Appendix. We get

$$
\begin{gathered}
\int_{\mathbb{R}} e^{-\frac{2 U(y)}{\varepsilon}} d y=\sqrt{\frac{\pi \varepsilon}{\omega_{-}}} e^{\frac{V}{\varepsilon}}\left[1+\frac{\varepsilon}{16 \omega_{-}^{3}}\left(\frac{5 U^{(3)}(-1)^{2}}{3}-\omega_{-} U^{(4)}(-1)\right)+\mathcal{O}\left(\varepsilon^{2}\right)\right], \\
\int_{\mathbb{R}} y e^{-\frac{2 U(y)}{\varepsilon}} d y=-\sqrt{\frac{\pi \varepsilon}{\omega_{-}}} e^{\frac{V}{\varepsilon}} \\
\times\left[1+\frac{U^{(3)}(-1)}{4 \omega_{-}^{2}} \varepsilon+\frac{\varepsilon}{16 \omega_{-}^{3}}\left(\frac{5 U^{(3)}(-1)^{2}}{3}-\omega_{-} U^{(4)}(-1)\right)+\mathcal{O}\left(\varepsilon^{2}\right)\right] .
\end{gathered}
$$


The relationships (55) and (57) follow from these formulae and the asymptotic expansion rule $\frac{1+a_{1} \varepsilon+\mathcal{O}\left(\varepsilon^{2}\right)}{1+a_{2} \varepsilon+\mathcal{O}\left(\varepsilon^{2}\right)}=1+\left(a_{1}-a_{2}\right) \varepsilon+\mathcal{O}\left(\varepsilon^{2}\right), a_{1}, a_{2} \in \mathbb{R}$.

The estimate for $b_{1}$ is obtained analogously to Lemma 4.1 with the help of Proposition 4.2. More precisely, we get

$$
\begin{aligned}
b_{1} & =-\frac{1+e^{-\lambda_{1} T}}{2} \frac{\int_{\mathbb{R}} y \Psi_{1}(y) d y}{\left\|\Psi_{0}\right\|_{\rho}^{2}} \frac{\left(\bar{\Psi}_{0}, \Psi_{1}\right)_{\rho}}{\left\|\Psi_{1}\right\|_{\rho}^{2}-e^{-\lambda_{1} T}\left(\bar{\Psi}_{1}, \Psi_{1}\right)_{\rho}} \\
& =-\frac{2\left(1+e^{-\lambda_{1} T}\right) \cdot \sqrt{\frac{\pi \varepsilon}{\omega_{+}}} e^{\frac{v}{\varepsilon}}(1+\mathcal{O}(\varepsilon)) \cdot \sqrt{\frac{\pi \varepsilon}{\omega_{-}}} e^{\frac{V}{\varepsilon}}(1+\mathcal{O}(\varepsilon))}{2 \sqrt{\frac{\pi \varepsilon}{\omega_{-}}} e^{\frac{V}{\varepsilon}}(1+\mathcal{O}(\varepsilon)) \cdot\left(\sqrt{\frac{\pi \varepsilon}{\omega_{+}}} e^{\frac{v}{\varepsilon}}(1+\mathcal{O}(\varepsilon))+e^{-\lambda_{1}^{\varepsilon} T} \sqrt{\frac{\pi \varepsilon}{\omega_{+}}} e^{\frac{v}{\varepsilon}}(1+\mathcal{O}(\varepsilon))\right)} \\
& =-1+\mathcal{O}(\varepsilon) .
\end{aligned}
$$

The combination of (55) and (56) leads to (58).

Let us compare the first term in (52) with $\eta^{Y}$. Lemma 4.2 states that $b_{0} \approx 1$ as $\varepsilon \rightarrow 0$. Recall that

$$
\lambda_{1} \approx \frac{\sqrt{\omega_{0} \omega_{+}}}{2 \pi} e^{-\frac{v}{\varepsilon}}
$$

and that the infinitesimal probabilities in Section 3 were defined by $\varphi=p e^{-\frac{V}{\varepsilon}}$ and $\psi=p e^{-\frac{v}{\varepsilon}}$, with $p, q>0$.

We now choose $p$ and $q$ so that Kramers' times for the diffusion and the reducing Markov chain coincide not only to exponential order, but in addition up to the leading subexponential pre-factors. If we set

$$
p=\frac{\sqrt{\omega_{0} \omega_{-}}}{2 \pi}, \quad q=\frac{\sqrt{\omega_{0} \omega_{+}}}{2 \pi}
$$

we also get

$$
\psi+\varphi \approx \lambda_{1}, \quad \psi-\varphi \approx \lambda_{1}
$$

to the leading subexponential pre-factor. This moreover implies

$$
b_{0}^{2} \frac{\left(\lambda_{1} T\right)^{2}}{\pi^{2}+\left(\lambda_{1} T\right)^{2}} \approx \frac{(\psi-\varphi)^{2} T^{2}}{\pi^{2}+(\psi+\varphi)^{2} T^{2}} .
$$

This correspondence is a sure temptation for suspecting that provided the remainder terms in (52) are small enough, the SPA coefficients of the diffusion and the Markov chain are close. This also strongly suggests that one could be able to relate asymptotically the optimal tuning rates for the diffusion and the reducing Markov chain.

This argument is in fact very common in the physics literature $[30,20,1]$ and is used to pass to a simpler two-state framework for the investigation of various dynamical properties of the diffusion in the small noise limit, especially in the context of stochastic resonance. This approach will now be shown to possibly have drastic side effects. In determining eigenvalues and Kramers' times we always had to take into account a multiplicative correction term of the type $1+\mathcal{O}(\varepsilon)$. The errors of order $\mathcal{O}(\varepsilon)$ in all the formulae we have derived reflect small random fluctuations of the diffusion near the metastable states of the potential. Neglecting these terms means neglecting 
the 'diffusive' nature of the diffusion. Although these fluctuations are small, they occur with high probability. This leads to very subtle drag effects in the potential wells' bottoms disturbing or even destroying the tuning picture the two-state reduction presents.

In Section 3 the optimal tuning rate for the Markov chain $Y^{\varepsilon, T}$ in the sense of the SPA coefficient was determined by

$$
\varepsilon=\frac{V+v}{2 \log T}\left(1+\frac{\log \left(\frac{\pi}{\sqrt{2 p q}} \sqrt{\frac{v}{V-v}}\right)}{\log T}+\mathcal{O}\left(\frac{1}{\log ^{2} T}\right)\right) .
$$

If the diffusion's tuning properties corresponding to the SPA coefficient were retained by the reducing Markov chain, we would expect a local maximum of the SPA coefficient $\eta^{X}(\cdot, T)$ at some point $\varepsilon(T) \approx \frac{V+v}{2 \log T}$. Let us consider $\alpha \mapsto \eta^{X}\left(\frac{\alpha}{\log T}, T\right)$ for large $T$ on the interval

$$
[v+\delta, \Delta], \quad \text { for some } \delta>0, \Delta>v+\delta .
$$

On the $\varepsilon$-scale this corresponds to shrinking intervals $\left[\frac{v+\delta}{\log T}, \frac{\Delta}{\log T}\right]$. We shall investigate, whether $\alpha \mapsto \eta^{X}\left(\frac{\alpha}{\log T}, T\right)$ possesses a local maximum for large $T$.

Theorem 4.1 Let $0<\delta<\frac{v}{3}$ and $\Delta>v+\delta$ be fixed. Let $\max _{x \in \mathbb{R}}\{U(x)-2 U(-x)\}<$ $V+v$. Then there exists $T_{0}>0$ such that for $T>T_{0}, \alpha \in[v+\delta, \Delta]$ we have

$$
\eta^{X}\left(\frac{\alpha}{\log T}, T\right)=\frac{4}{\pi^{2}}\left(1+\frac{U^{(3)}(-1)}{2 \omega_{-}^{2}} \frac{\alpha}{\log T}\right)+\mathcal{O}\left(\frac{1}{\log ^{2} T}\right) .
$$

Remark 4.1 Geometrically, the condition $\max _{x \in \mathbb{R}}\{U(x)-2 U(-x)\}<V+v$ may be seen to express the fact that the potential is not too asymmetric outside of the wells.

Proof: The proof consists in expanding (52) as $T \rightarrow \infty$ and estimating the remainder terms.

First, we note that in order to apply Lemma 4.1 the noise parameter $\varepsilon$ must satisfy $\varepsilon \in\left[\frac{v+\delta}{\log T}, \varepsilon_{0}\right]$ for some $\varepsilon_{0}>0$. It is clear that to verify this condition it is enough to take $T_{0}>e^{\Delta / \varepsilon_{0}}$.

Consider $\eta^{X}\left(\frac{\alpha}{\log T}, T\right)$ for $T>0$. The factor $b_{0}$ in the leading term of $\eta^{X}$ is expanded with the help of Lemma 4.2.

On the interval $\left[\frac{v+\delta}{\log T}, \frac{\Delta}{\log T}\right]$ with some constant $C>0$ we estimate

$$
\left(\lambda_{1} T\right)^{2} \geq C\left(e^{-\frac{v}{\varepsilon}} T\right)^{2} \geq C\left(T^{1-\frac{v}{v+\delta}}\right)^{2}=C T^{\frac{2 \delta}{v+\delta}} .
$$

This results in

$$
\frac{\left(\lambda_{1} T\right)^{2}}{\pi^{2}+\left(\lambda_{1} T\right)^{2}}=1-\frac{\pi^{2}}{\pi^{2}+\left(\lambda_{1} T\right)^{2}}=1+\mathcal{O}\left(\frac{1}{\left(\lambda_{1} T\right)^{2}}\right)=1+\mathcal{O}\left(\frac{1}{T^{\frac{2 \delta}{v+\delta}}}\right) .
$$

Hence, we obtain the expansion of the leading term of (52)

$$
\frac{4}{\pi^{2}} \frac{b_{0}^{2}\left(\lambda_{1} T\right)^{2}}{\pi^{2}+\left(\lambda_{1} T\right)^{2}}=\frac{4}{\pi^{2}}\left(1+\frac{U^{(3)}(-1)}{2 \omega_{-}^{2}} \frac{\alpha}{\log T}\right)+\mathcal{O}\left(\frac{1}{\log ^{2} T}\right) .
$$


It remains to estimate the terms $\frac{4\left(b_{1}-b_{0}\right)^{2}}{\pi^{2}+\left(\lambda_{1} T\right)^{2}}$ and $\left|r_{3}\right|$. Analogously to (60), the first one is of the order $T^{-\frac{2 \delta}{v+\delta}} \log ^{-2} T$ on $\alpha \in[v+\delta, \Delta]$ as $T \rightarrow \infty$ since $\left(b_{1}-b_{0}\right)^{2}=\mathcal{O}\left(\frac{1}{\log ^{2} T}\right)$.

Consider the third term given by (53):

$$
\left|r_{3}(\varepsilon, T)\right| \leq 8\left|s^{X}(\varepsilon, T)\right|\left|r_{2}(\varepsilon, T)\right|+4\left|r_{2}(\varepsilon, T)\right|^{2},
$$

where

$$
\left|s^{X}(\varepsilon, T)\right|=\left(\frac{1}{\pi^{2}} \frac{b_{0}^{2}\left(\lambda_{1} T\right)^{2}}{\pi^{2}+\left(\lambda_{1} T\right)^{2}}+\frac{4\left(b_{1}-b_{0}\right)^{2}}{\pi^{2}+\left(\lambda_{1} T\right)^{2}}\right)^{\frac{1}{2}} \leq \frac{1}{2}
$$

for $T \geq T_{0}, \frac{v+\delta}{\log T} \leq \varepsilon \leq \frac{\Delta}{\log T}$.

Let us estimate $\left|r_{2}(\varepsilon, T)\right|$. Lemma 4.1 states that

$$
\left|r_{2}(\varepsilon, T)\right| \leq \frac{6}{M T} \frac{\left[\int_{\mathbb{R}} e^{-\frac{2}{\varepsilon}(2 U(-y)-U(y))} d y\right]^{\frac{1}{2}}\left[\int_{\mathbb{R}} y e^{-\frac{2 U(y)}{\varepsilon}} d y\right]^{\frac{1}{2}}}{\int_{\mathbb{R}} e^{-\frac{2 U(y)}{\varepsilon}} d y} .
$$

Assume that $\max _{x \in \mathbb{R}}\{U(x)-2 U(-x)\}<\kappa$, where $\kappa$ is a positive number. Obviously, $\kappa>V-\frac{v}{2}>0$, since

$$
\max _{x \in \mathbb{R}}\{U(x)-2 U(-x)\} \geq U(1)-2 U(-1)=V-\frac{v}{2} .
$$

Then, using Laplace's method we obtain for some $C>0$ independent of $\varepsilon \leq \varepsilon_{0}$

$$
\left|r_{2}(\varepsilon, T)\right| \leq C \frac{e^{\frac{\kappa}{2 \varepsilon}} e^{\frac{V}{2 \varepsilon}}}{T e^{\frac{V}{\varepsilon}}}=C \frac{e^{\frac{\kappa-V}{2 \varepsilon}}}{T}
$$

If we choose $\kappa=V+v$ and $\delta \in\left(0, \frac{v}{3}\right)$, then the inequality $\frac{v+2 \delta}{2(v+\delta)}>\frac{2 \delta}{v+\delta}$ entails that

$$
\max _{\alpha \in[v+\delta, \Delta]}\left|r_{2}\left(\frac{\alpha}{\log T}, T\right)\right| \leq C \frac{1}{T^{1-\frac{v}{2(v+\delta)}}}=o\left(\frac{1}{T^{\frac{2 \delta}{v+\delta}}}\right), \quad \delta \in\left(0, \frac{v}{3}\right) .
$$

Thus, the remainder terms in (52) are polynomially small and of the order $T^{-\frac{2 \delta}{v+\delta}} \log ^{-2} T$. This completes the proof.

As we see, the form of the tuning curve for the SPA coefficient crucially depends on the sign of $U^{(3)}(-1)$. If $U^{(3)}(-1)>0$, the tuning curve increases in $\alpha$ and does not have a local maximum on the interval $\left[\frac{v+\delta}{\log T}, \frac{\Delta}{\log T}\right]$. If $U^{(3)}(-1)<0$ then $\eta^{X}$ decreases and does not have a local maximum either.

Moreover, depending on the sign of $U^{(3)}(-1)$, the resonance curve is either greater or less than $\frac{4}{\pi^{2}}$. However on the resonance interval, one can see that the SPA coefficient is near the maximal value of its Markov chain counterpart. This means, that amplification occurs, but an optimal tuning rate cannot be determined. Especially, the Markov chain behaviour is not shared by the diffusion, and the optimal tuning rate by the chain is not asymptotically equal to an optimal tuning rate for the diffusion, in which sense ever the latter may exist. 
The observed subtle drag effect may be interpreted in the following way. If $U^{(3)}(-1)<$ 0 , the potential in the deep well slightly leans towards the shallow one. Therefore in the $\varepsilon$-window considered increasing the noise intensity tends to reduce the averaged overall amplitude of the motion.

In case $U^{(3)}(-1)>0$, the outward leaning of the potential in its global maximum increases the averaged overall amplitude of the random motion with increasing noise intensity.

\subsection{Optimal tuning for the modified SPA coefficient.}

Stochastic resonance is an inter-well and not an intra-well effect. Given our experience gained in the previous subsection, we now suppress fluctuations near the potential minima and take into account only big hoppings between the wells. In this modified setting, we shall now show that the behaviour of the reducing Markov chain is correctly retained asymptotically in the small noise limit.

In order to cut off small random fluctuations near -1 and 1 we define a function

$$
g(x)=\left\{\begin{aligned}
x, & x \in\left(-\infty, x_{1}\right] \cup\left[x_{2}, y_{1}\right] \cup\left[y_{2}, \infty\right), \\
-1, & x \in\left[x_{1}, x_{2}\right], \\
1, & x \in\left[y_{1}, y_{2}\right],
\end{aligned}\right.
$$

where $x_{1}<-1<x_{2}<0$ are such that $U\left(x_{1}\right)=U\left(x_{2}\right)=-\frac{V}{4}$, and $0<y_{1}<1<y_{2}$ are such that $U\left(y_{1}\right)=U\left(y_{2}\right)=-\frac{v}{4}$.

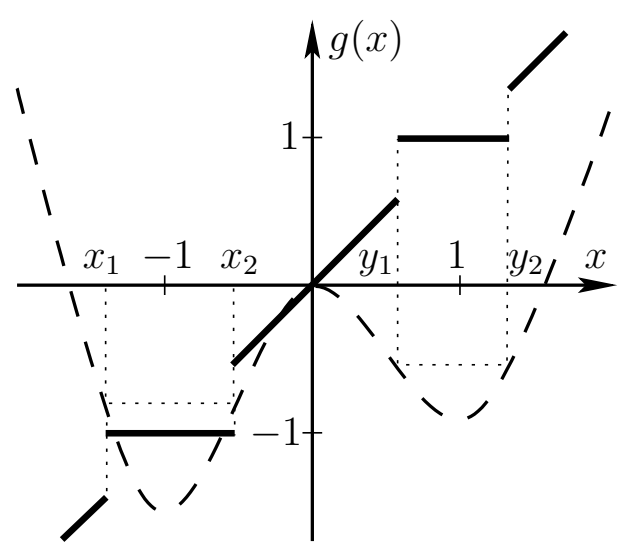

Fig. 13: Function $g$ designed to cut off small fluctuations.

For $\varepsilon, T>0$ we consider the modified SPA coefficient

$$
\widetilde{\eta}^{X}(\varepsilon, T)=\left|\int_{0}^{1} \mathbf{E}_{\mu} g\left(X_{2 T s}^{\varepsilon, T}\right) e^{2 \pi i s} d s\right|^{2} .
$$

By inspection of the steps in the calculations of subsection 4.3, replacing $x$ with $g(x)$ if necessary, we obtain a formula for $\widetilde{\eta}^{X}$ which is analogous to (52):

$$
\widetilde{\eta}^{X}(\varepsilon, T)=\frac{4}{\pi^{2}} \frac{\widetilde{b}_{0}^{2}\left(\lambda_{1} T\right)^{2}}{\pi^{2}+\left(\lambda_{1} T\right)^{2}}+4 \frac{\left(\widetilde{b}_{1}-\widetilde{b}_{0}\right)^{2}}{\pi^{2}+\left(\lambda_{1} T\right)^{2}}+\widetilde{r}_{3}(\varepsilon, T),
$$


where

$$
\begin{aligned}
\widetilde{b}_{0} & =\frac{\int_{\mathbb{R}} g(y) e^{-\frac{2 U(y)}{\varepsilon}} d y}{\int_{\mathbb{R}} e^{-\frac{2 U(y)}{\varepsilon}} d y}, \\
\widetilde{b}_{1} & =-\frac{1+e^{-\lambda_{1} T}}{2} \frac{\int_{\mathbb{R}} g(y) \Psi_{1}(y) d y}{\left\|\Psi_{0}\right\|_{\rho}^{2}} \frac{\left(\bar{\Psi}_{0}, \Psi_{1}\right)_{\rho}}{\left\|\Psi_{1}\right\|_{\rho}^{2}-e^{-\lambda_{1} T}\left(\bar{\Psi}_{1}, \Psi_{1}\right)_{\rho}}, \\
\widetilde{r}_{3}(\varepsilon, T) & =4\left|\widetilde{r}_{2}(\varepsilon, T)\right|^{2}+8 \operatorname{Re}\left(\widetilde{s}^{X}(\varepsilon, T) \widetilde{r}_{2}^{*}(\varepsilon, T)\right), \\
\widetilde{s}^{X}(\varepsilon, T) & =\frac{i}{\pi} \widetilde{b}_{0}+\frac{1}{\pi i-\lambda_{1} T} \widetilde{b}_{1}, \\
\left|\widetilde{r}_{2}(\varepsilon, T)\right| & \leq \frac{6}{M T}\left(\int_{\mathbb{R}} e^{-\frac{2 U(y)}{\varepsilon}} d y\right)^{-1}\left\|e^{-\frac{2 \bar{U}}{\varepsilon}}\right\|_{\rho} \cdot\left\|g e^{-\frac{2 U}{\varepsilon}}\right\|_{\rho} .
\end{aligned}
$$

It turns out that the factors $\widetilde{b}_{0}$ and $b_{0}$ have quite different asymptotics. Hence the modified SPA coefficient $\widetilde{\eta}^{X}(\varepsilon, T)$ has a local maximum close to the corresponding one for the Markov chain.

Lemma 4.3 There is $\varepsilon_{0}>0$ such that for $\varepsilon \leq \varepsilon_{0}, T>0$ we have

$$
\begin{aligned}
& \widetilde{b}_{0}=-1+2 \sqrt{\frac{\omega_{-}}{\omega_{+}}} e^{-\frac{V-v}{\varepsilon}}(1+\mathcal{O}(\varepsilon)) \geq-1, \\
& \widetilde{b}_{1}=-1+\mathcal{O}(\varepsilon),
\end{aligned}
$$

and, consequently,

$$
\begin{aligned}
& \widetilde{b}_{0}^{2}=1-4 \sqrt{\frac{\omega_{-}}{\omega_{+}}} e^{-\frac{V-v}{\varepsilon}}(1+\mathcal{O}(\varepsilon)) \leq 1 \\
& \left(\widetilde{b}_{1}-\widetilde{b}_{0}\right)^{2}=\mathcal{O}\left(\varepsilon^{2}\right) .
\end{aligned}
$$

Proof: Consider $\widetilde{b}_{0}$ for small $\varepsilon$ and use Laplace's method to obtain (62):

$$
\begin{aligned}
\widetilde{b}_{0} & =\frac{\int_{\mathbb{R}} g(y) e^{-\frac{2 U(y)}{\varepsilon}} d y}{\int_{\mathbb{R}} e^{-\frac{2 U(y)}{\varepsilon}} d y} \\
& =\frac{\left(-\int_{x_{1}}^{x_{2}}+\int_{y_{1}}^{y_{2}}\right) e^{-\frac{2 U(y)}{\varepsilon}} d y+\left(\int_{-\infty}^{x_{1}}+\int_{x_{2}}^{y_{1}}+\int_{y_{2}}^{\infty}\right) y e^{-\frac{2 U(y)}{\varepsilon}} d y}{\left(\int_{x_{1}}^{x_{2}}+\int_{y_{1}}^{y_{2}}\right) e^{-\frac{2 U(y)}{\varepsilon}} d y+\left(\int_{-\infty}^{x_{1}}+\int_{x_{2}}^{y_{1}}+\int_{y_{2}}^{\infty}\right) e^{-\frac{2 U(y)}{\varepsilon}} d y} \\
& =-\frac{\left(\int_{x_{1}}^{x_{2}}+\int_{y_{1}}^{y_{2}}\right) e^{-\frac{2 U(y)}{\varepsilon}} d y-2 \int_{y_{1}}^{y_{2}} e^{-\frac{2 U(y)}{\varepsilon}} d y+\mathcal{O}\left(e^{\frac{V}{2 \varepsilon}}\right)}{\left(\int_{x_{1}}^{x_{2}}+\int_{y_{1}}^{y_{2}}\right) e^{-\frac{2 U(y)}{\varepsilon}} d y+\mathcal{O}\left(e^{\frac{V}{2 \varepsilon}}\right)} \\
& =-1+\frac{2 \sqrt{\frac{\pi \varepsilon}{\omega_{+}}} e^{\frac{v}{\varepsilon}}(1+\mathcal{O}(\varepsilon))}{\sqrt{\frac{\pi \varepsilon}{\omega_{-}}} e^{\frac{V}{\varepsilon}}(1+\mathcal{O}(\varepsilon))}=-1+2 \sqrt{\frac{\omega_{-}}{\omega_{+}}} e^{-\frac{V-v}{\varepsilon}}(1+\mathcal{O}(\varepsilon)) .
\end{aligned}
$$

For the third equation we used our hypothesis concerning the cutoff levels, and the inequality $V-v<\frac{V}{2}$ which follows from $(\mathbf{M})$. 
Formulae (63) and (65) are obtained analogously to Lemma 4.2. Expression (64) follows directly from (62).

Theorem 4.2 Let $\max _{x \in \mathbb{R}}\{U(x)-2 U(-x)\}<V+v$ and $0<\delta<\frac{v}{3}$. Then for any $1<\gamma<\frac{V+v}{2(v+\delta)}$ there exists $T(\gamma)$ such that for $T>T(\gamma)$ the modified SPA coefficient $\varepsilon \mapsto \widetilde{\eta}^{X}(\varepsilon, T)$ has a local maximum on $\left[\gamma^{-1} \frac{V+v}{2 \log T}, \gamma \frac{V+v}{2 \log T}\right]$. The optimal tuning rate $\varepsilon(T)$ is exponentially equivalent to $\frac{V+v}{2 \log T}$ in the limit $T \rightarrow \infty$.

Proof: To show that $\varepsilon \mapsto \widetilde{\eta}^{X}(\varepsilon, T)$ given by (61) has a local maximum we consider it at the three points

$$
\varepsilon_{1}(T)=\frac{V+v}{2 \log T}, \quad \varepsilon_{2}(T)=\gamma \frac{V+v}{2 \log T}, \text { and } \varepsilon_{3}(T)=\gamma^{-1} \frac{V+v}{2 \log T} .
$$

Since $\gamma>1$, we have $\varepsilon_{3}(T)<\varepsilon_{1}(T)<\varepsilon_{2}(T)$ (see Fig. 14).

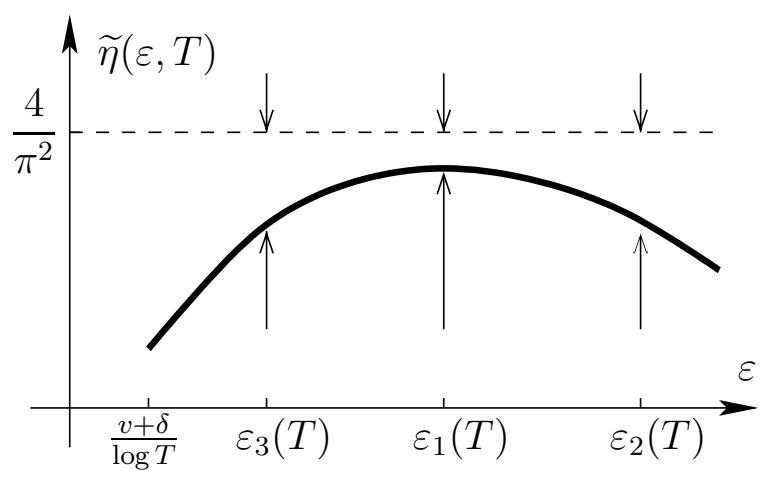

Fig. 14: The modified SPA coefficient $\widetilde{\eta}^{X}(\varepsilon, T)$ at the points $\varepsilon_{i}(T), i=1,2,3$, defined in (66).

Consider the leading term of $\widetilde{\eta}^{X}\left(\varepsilon_{1}(T), T\right)$ for $T \rightarrow \infty$. The factor $\widetilde{b}_{0}^{2}$ is given by Lemma 4.3. Expanding the factor $\frac{\left(\lambda_{1} T\right)^{2}}{\pi^{2}+\left(\lambda_{1} T\right)^{2}}$ as $T \rightarrow \infty$ gives:

$$
\begin{aligned}
\frac{4}{\pi^{2}} \frac{\widetilde{b}_{0}^{2}\left(\lambda_{1} T\right)^{2}}{\pi^{2}+\left(\lambda_{1} T\right)^{2}} & =\frac{4}{\pi^{2}}\left(1-4 \sqrt{\frac{\omega_{-}}{\omega_{+}}} T^{-2 \frac{V-v}{V+v}}\left(1+\mathcal{O}\left(\log ^{-1} T\right)\right)\right) \\
& \times\left(1-\frac{4 \pi^{4}}{\omega_{0} \omega_{+}} T^{-2 \frac{V-v}{V+v}}\left(1+\mathcal{O}\left(\log ^{-1} T\right)\right)\right) \\
& =\frac{4}{\pi^{2}}-\frac{16}{\pi^{2}}\left(\sqrt{\frac{\omega_{-}}{\omega_{+}}}+\frac{\pi^{4}}{\omega_{0} \omega_{+}}\right) T^{-2 \frac{V-v}{V+v}}\left(1+\mathcal{O}\left(\log ^{-1} T\right)\right) .
\end{aligned}
$$

Analogously to Theorem 4.1 one shows that the remainder terms in (61) are of the order $T^{-2 \frac{V-v}{V+v}} \log ^{-2} T$. This yields that

$$
\widetilde{\eta}^{X}\left(\varepsilon_{1}(T), T\right)=\frac{4}{\pi^{2}}-\frac{16}{\pi^{2}}\left(\sqrt{\frac{\omega_{-}}{\omega_{+}}}+\frac{\pi^{4}}{\omega_{0} \omega_{+}}\right) \frac{1}{T^{2 \frac{V-v}{V+v}}}\left(1+\mathcal{O}\left(\frac{1}{\log T}\right)\right) .
$$


Analogously, we consider the modified SPA coefficient at $\varepsilon_{2}(T)$ and $\varepsilon_{3}(T)$ to obtain

$$
\widetilde{\eta}^{X}\left(\varepsilon_{2}(T), T\right)=\frac{4}{\pi^{2}}-\frac{16}{\pi^{2}} \sqrt{\frac{\omega_{-}}{\omega_{+}}} \frac{1}{T^{2} \frac{V-v}{\gamma(V+v)}}\left(1+\mathcal{O}\left(\frac{1}{\log T}\right)\right),
$$

and

$$
\widetilde{\eta}^{X}\left(\varepsilon_{3}(T), T\right)=\frac{4}{\pi^{2}}-\frac{16 \pi^{2}}{\omega_{0} \omega_{+}} \frac{1}{T^{2 \frac{V+v-2 \gamma v}{V+v}}}\left(1+\mathcal{O}\left(\frac{1}{\log T}\right)\right) .
$$

This entails that for $T(\gamma)$ large enough and $T>T(\gamma)$ we have

$$
\begin{aligned}
& \widetilde{\eta}^{X}\left(\varepsilon_{1}(T), T\right)>\widetilde{\eta}^{X}\left(\varepsilon_{2}(T), T\right) \quad \text { and } \\
& \widetilde{\eta}^{X}\left(\varepsilon_{1}(T), T\right)>\widetilde{\eta}^{X}\left(\varepsilon_{3}(T), T\right)
\end{aligned}
$$

since $V-v>\frac{1}{\gamma}(V-v)$ and $V-v>V+v-2 \gamma v$ for $\gamma>1$.

Since the differences between the values taken are of higher order than the bounds for the remainder terms, this completes the proof.

Let us finish with some remarks concerning the dependence of the optimal tuning rate on the geometry of the potential $U$. We have seen in Section 3 that for some values of the pre-factors $p$ and $q$ and half-period $T$ the tuning curve vanishes at certain noise levels or is monotonically increasing, see Proposition 3.4. We do not observe such a phenomenon in the present setting since we consider the SPA coefficient of the diffusion in the small noise and large period limits. Recall that the Markov chain SPA coefficient vanishes at $\widehat{\varepsilon}=(V-v) / \log \left(\frac{p}{q}\right)$ which is a positive number independent of $\varepsilon$ and $T$. Of course, it can happen that $\eta^{X}$ or $\widetilde{\eta}^{X}$ vanishes for some noise intensity. However, our approach describes neither this effect nor monotonicity of $\widetilde{\eta}^{X}$. The reason is this: in the small noise limit considered here we are outside of the domains of parameter space for which this behaviour is exhibited.

\section{A Laplace's method}

Consider the integral

$$
I(\varepsilon)=\int_{a}^{b} e^{-\frac{2 U(x)}{\varepsilon}} w(x) d x
$$

in which $a, b \in[-\infty,+\infty], U$ and $w$ are smooth functions on $\mathbb{R}, \varepsilon>0$. If $U$ has a finite number of minima, we may break up the integral (67) into a finite number of integrals so that in each interval $U$ reaches its minimum at one of the end-points and at no other point. Accordingly, we shall assume that $U$ reaches its minimum at $x=a$ and that $U(x)>U(a), a<x \leq b$. We now precisely formulate the Laplace's approximation theorem following [31, Chapters 7,9].

Theorem A.1 Let $a \in \mathbb{R}, b \in \mathbb{R} \cup\{+\infty\}, a<b$. Let $U: \mathbb{R} \rightarrow \mathbb{R}$ be differentiable, and $w: \mathbb{R} \rightarrow \mathbb{R}$ or $\mathbb{C}$ be measurable.

Suppose in addition that 
i) the minimum of $U$ is attained only at a;

ii) $U^{\prime}$ and $w$ are continuous in a neighbourhood of a;

iii) as $x \downarrow$ a,

$U(x)=U(a)+P(x-a)^{\mu}+\mathcal{O}\left((x-a)^{\mu+1}\right), \quad w(x)=Q(x-a)^{\lambda-1}+\mathcal{O}\left((x-a)^{\lambda}\right)$,

and the first of these relations is differentiable. Here $P, \mu$ and $\lambda$ are positive constants, and $Q$ is a real or complex constant.

iv)

$$
I(\varepsilon)=\int_{a}^{b} e^{-\frac{2 U(x)}{\varepsilon}} w(x) d x
$$

converges absolutely throughout its range for all sufficiently small $\varepsilon$.

Then

$$
I(\varepsilon)=\frac{Q}{\mu} \Gamma\left(\frac{\lambda}{\mu}\right)\left(\frac{\varepsilon}{2 P}\right)^{\frac{\lambda}{\mu}} e^{-\frac{2 U(a)}{\varepsilon}}\left(1+\mathcal{O}\left(\varepsilon^{\frac{1}{\mu}}\right)\right) .
$$

If the asymptotic expansions in ascending powers of $x-a$ exist for $U$ and $w$, the expansion of the integral $I(\varepsilon)$ can be also obtained. Although there is no general formula for this expansion, we determine its first three terms.

Theorem A.2 Let conditions (i), (ii) and (iv) of Theorem A.1 be satisfied and the expansions

$$
\begin{aligned}
& U(x)=U(a)+\sum_{s=0}^{n-1} p_{s}(x-a)^{\mu+s}+\mathcal{O}\left((x-a)^{\mu+n}\right), \\
& w(x)=\sum_{s=0}^{n-1} q_{s}(x-a)^{\lambda-1+s}+\mathcal{O}\left((x-a)^{\lambda+n}\right)
\end{aligned}
$$

hold. Suppose that $p_{0} \neq 0, q_{0} \neq 0$. Then

$$
I(\varepsilon)=e^{-\frac{2 U(a)}{\varepsilon}}\left[\sum_{s=0}^{n-1} \Gamma\left(\frac{\lambda+s}{\mu}\right) a_{s}\left(\frac{\varepsilon}{2}\right)^{\frac{\lambda+s}{\mu}}+\mathcal{O}\left(\varepsilon^{\frac{\lambda+n}{\mu}}\right)\right],
$$

where

$$
\begin{aligned}
& a_{0}=\frac{q_{0}}{\mu p_{0}^{\lambda / \mu}} \\
& a_{1}=\left\{\frac{q_{1}}{\mu}-\frac{(\lambda+1) p_{1} q_{0}}{\mu^{2} p_{0}}\right\} \frac{1}{p_{0}^{(\lambda+1) / \mu}}, \\
& a_{2}=\left[\frac{q_{2}}{\mu}-\frac{(\lambda+2) p_{1} q_{1}}{\mu^{2} p_{0}}+\left\{(\lambda+\mu+2) p_{1}^{2}-2 \mu p_{0} p_{2}\right\} \frac{(\lambda+2) q_{0}}{2 \mu^{3} p_{0}^{2}}\right] \frac{1}{p_{0}^{(\lambda+2) / \mu}} .
\end{aligned}
$$


Let us apply Theorems A.1 and A.2 to the double-well potential $U$ from Section 4 to find the asymptotics of the integral $\int_{\mathbb{R}} e^{-\frac{2 U(x)}{\varepsilon}} d x$ for $\varepsilon \rightarrow 0$.

The function $U$ is supposed to be infinitely differentiable and to possess a unique global minimum at -1 such that $U(-1)=-\frac{V}{2}$. We break the interval $(-\infty,+\infty)$ into two intervals $(-\infty,-1]$ and $[-1,+\infty)$, and note that

$$
\begin{aligned}
\int_{\mathbb{R}} e^{-\frac{2 U(x)}{\varepsilon}} d x & =\int_{-\infty}^{-1} e^{-\frac{2 U(x)}{\varepsilon}} d x+\int_{-1}^{\infty} e^{-\frac{2 U(x)}{\varepsilon}} d x \\
& =\int_{-1}^{+\infty} e^{-\frac{2 U(x)}{\varepsilon}} d x+\int_{1}^{+\infty} e^{-\frac{2 \bar{U}(x)}{\varepsilon}} d x
\end{aligned}
$$

where $\bar{U}(x)=U(-x), x \in \mathbb{R}$. Both integrals in the last line of (70) satisfy the conditions of Theorem A.2. To determine the coefficients $p_{k}, k=0,1,2$, we expand $U$ near -1 and $\bar{U}$ near 1 to get

$$
\begin{aligned}
& U(x)=-\frac{V}{2}+\frac{\omega_{-}}{2}(x+1)^{2}+\frac{U^{(3)}(-1)}{6}(x+1)^{3}+\frac{U^{(4)}(-1)}{24}(x+1)^{4}+\mathcal{O}\left((x+1)^{5}\right), \\
& \bar{U}(x)=-\frac{V}{2}+\frac{\omega_{-}}{2}(x-1)^{2}-\frac{U^{(3)}(-1)}{6}(x-1)^{3}+\frac{U^{(4)}(-1)}{24}(x-1)^{4}+\mathcal{O}\left((x-1)^{5}\right) .
\end{aligned}
$$

Thus, $\mu=2, \lambda=1, q_{0}=1$ and $q_{k}=0, k \geq 1$. A direct application of (69) and (68) yields

$$
\begin{aligned}
& \int_{-1}^{+\infty} e^{-\frac{2 U(x)}{\varepsilon}} d x=e^{\frac{V}{\varepsilon}} \frac{1}{2} \sqrt{\frac{\pi \varepsilon}{\omega_{-}}} \\
& \times\left[1-\frac{U^{(3)}(-1)}{3 \omega_{-}^{3 / 2} \sqrt{\pi}} \sqrt{\varepsilon}+\frac{1}{16 \omega_{-}^{3}}\left(\frac{5 U^{(3)}(-1)^{2}}{3}-\omega_{-} U^{(4)}(-1)\right) \varepsilon+\mathcal{O}\left(\varepsilon^{3 / 2}\right)\right] \\
& \int_{1}^{+\infty} e^{-\frac{2 \bar{U}(x)}{\varepsilon}} d x=e^{\frac{V}{\varepsilon}} \frac{1}{2} \sqrt{\frac{\pi \varepsilon}{\omega_{-}}} \\
& \times\left[1+\frac{U^{(3)}(-1)}{3 \omega_{-}^{3 / 2} \sqrt{\pi}} \sqrt{\varepsilon}+\frac{1}{16 \omega_{-}^{3}}\left(\frac{5 U^{(3)}(-1)^{2}}{3}-\omega_{-} U^{(4)}(-1)\right) \varepsilon+\mathcal{O}\left(\varepsilon^{3 / 2}\right)\right]
\end{aligned}
$$

and consequently

$$
\int_{\mathbb{R}} e^{-\frac{2 U(x)}{\varepsilon}} d x=e^{\frac{V}{\varepsilon}} \sqrt{\frac{\pi \varepsilon}{\omega_{-}}}\left[1+\frac{1}{16 \omega_{-}^{3}}\left(\frac{5 U^{(3)}(-1)^{2}}{3}-\omega_{-} U^{(4)}(-1)\right) \varepsilon+\mathcal{O}\left(\varepsilon^{3 / 2}\right)\right] .
$$

The error term $\mathcal{O}\left(\varepsilon^{3 / 2}\right)$ is in fact of order $\varepsilon^{2}$, since due to the infinite differentiability of $U$ all terms $a_{k}$ with odd indices $k$ vanish. This variant of the asymptotics is used in Lemma 4.2. The less exact asymptotics

$$
\int_{\mathbb{R}} e^{-\frac{2 U(x)}{\varepsilon}} d x=e^{\frac{V}{\varepsilon}} \sqrt{\frac{\pi \varepsilon}{\omega_{-}}}(1+\mathcal{O}(\varepsilon))
$$


is also used.

Analogously, one evaluates the integral

$$
\begin{aligned}
& \int_{\mathbb{R}} x e^{-\frac{2 U(x)}{\varepsilon}} d x=\int_{-1}^{\infty} x e^{-\frac{2 U(x)}{\varepsilon}} d x-\int_{1}^{\infty} x e^{-\frac{2 \bar{U}(x)}{\varepsilon}} d x= \\
& -e^{\frac{V}{\varepsilon}} \sqrt{\frac{\pi \varepsilon}{\omega_{-}}}\left[1+\left\{\frac{U^{(3)}(-1)}{4 \omega_{-}^{2}}+\frac{1}{16 \omega_{-}^{3}}\left(\frac{5 U^{(3)}(-1)^{2}}{3}-\omega_{-} U^{(4)}(-1)\right)\right\} \varepsilon+\mathcal{O}\left(\varepsilon^{2}\right)\right] .
\end{aligned}
$$

\section{References}

[1] Anishchenko, V. S., Neiman, A. B., Moss, F., and Schimansky-Geier, L. Stochastic resonance: noise-enhanced order. Physics-Uspekhi 42, 1 (1999), $7-36$.

[2] Benzi, R., Parisi, G., Sutera, A., and Vulpiani, A. The mechanism of stochastic resonance. J. Phys. A 14 (1981), 453-457.

[3] Benzi, R., Parisi, G., Sutera, A., And Vulpiani, A. Stochastic resonance in climatic changes. Tellus 34 (1982), 10-16.

[4] Benzi, R., Parisi, G., Sutera, A., And Vulpiani, A. A theory of stochastic resonance in climatic change. SIAM J. Appl. Math. 43 (1983), 563-578.

[5] Berglund, N., And Gentz, B. A sample-paths approach to noise-induced synchronization: Stochastic resonance in a double-well potential. Preprint No. 627, Weierstraß-Institut für Angewandte Analysis und Stochastik (WIAS), Berlin, 2001.

[6] Berglund, N., And Gentz, B. Beyond the Fokker-Planck equation: Pathwise control of noisy bistable systems. Preprint No. 688, Weierstraß-Institut für Angewandte Analysis und Stochastik (WIAS), Berlin, 2001.

[7] Berglund, N., And Gentz, B. Metastability in simple climate models: Pathwise analysis of slowly driven Langevin equations. Preprint No. 696, WeierstraßInstitut für Angewandte Analysis und Stochastik (WIAS), Berlin, 2001.

[8] Bovier, A., Eckhoff, M., Gayrard, V., And Klein, M. Metastability and low lying spectra in reversible Markov chains. Preprint No. 600, WeierstraßInstitut für Angewandte Analysis und Stochastik (WIAS), Berlin, 2000. To appear in Comm. Math. Phys.

[9] Bovier, A., Eckhoff, M., Gayrard, V., And Klein, M. Metastability and small eigenvalues in Markov chains. J. Phys. A 33 (2000), L447-L451.

[10] Bovier, A., Eckhoff, M., Gayrard, V., And Klein, M. Metastability in stochastic dynamics of disordered mean-field models. Probab. Theory Related Fields 119, 1 (2001), 99-161. 
[11] Budyko, M. I. The effect of solar radiation variations on the climate of the earth. Tellus 21 (1969), 611-619.

[12] Claussen, M., Kubatzki, C., Brovkin, V., Ganopolski, A., Hoelzmann, P., AND PACHUR, H.-J. Simulation of an abrupt change in Saharan vegetation in the mid-Holocene. Geophysical Research Letters 26 (July 1999), 2037+.

[13] Dykman, M. I., Velikovich, A. L., Golubev, G. P., Luchinskit, D. G., And Tsuprikov, S. V. Stochastic resonance in an all-optical passive bistable system. Journal of Experimental and Theoretical Physics Letters 53 (Feb. 1991), 193-197.

[14] Eckmann, J.-P., And Thomas, L. E. Remarks on stochastic resonance. J. Phys. A 15 (1982), 261-266.

[15] Egorov, Y. V., And Shubin, M. A. Foundations of the classical theory of partial differential equations. Springer, Berlin, 1998.

[16] Fischer, A., Schütte, C., Deuflhard, P., and Cordes, F. Hierarchial uncoupling-coupling of metastable conformations. Preprint, FU Berlin, 2001.

[17] Freidlin, M. I. Quasi-deterministic approximation, metastability and stochastic resonance. Physica D 137, 3-4 (2000), 333-352.

[18] Freidlin, M. I., AND Wentzell, A. D. Random perturbations of dynamical systems, second ed., vol. 260 of Grundlehren der Mathematischen Wissenschaften. Springer, New York, NY, 1998.

[19] Freund, J. A., Kienert, J., Schimansky-Geier, L., Beisner, B., Neiman, A., Russell, D. F., Yakusheva, T., And Moss, F. Behavioral stochastic resonance: How a noisy army betrays its outpost. Physical Review E 63 (Mar. 2001), 031910+.

[20] Gammaitoni, L., Hänggi, P., Jung, P., and Marchesoni, F. Stochastic resonance. Reviews of Modern Physics 70 (Jan. 1998), 223-287.

[21] Ganopolski, A., And Rahmstorf, S. Abrupt glacial climate changes due to stochastic resonance. Physical Review Letters 88, 3 (Jan. 2001), 038501+.

[22] Herrmann, S., And Imkeller, P. Barrier crossings characterize stochastic resonance. To appear in 'Stochastics and Dynamics', 2002.

[23] Hibbs, A. D., Singsaas, A. L., Jacobs, E. W., Bulsara, A. R., BeKkedahl, J., AND Moss, F. Stochastic resonance in a superconducting loop with a Josephson junction. Journal of Applied Physics 77 (Mar. 1995), 2582-2590.

[24] Huisinga, W., Meyn, S., And Schütte, C. Phase Transitions \& Metastability in Markovian and Molecular Systems. submitted, 2002. 
[25] ImKelleR, P. Energy balance models - viewed from stochastic dynamics. In Stochastic climate models. Proceedings of a workshop, Chorin, Germany, Summer 1999. (Basel, 2001), Imkeller, P. et al., Eds., vol. 49 of Prog. Probab., Birkhäuser, pp. 213-240.

[26] Ivey, C., Apkarian, A. V., And Chialvo, D. R. Noise-induced tuning curve changes in mechanoreceptors. J. Neurophysiol. 79 (1998), 1879-1890.

[27] Khas'minskiJ, R. Z. Stochastic stability of differential equations. Monographs and Textbooks on Mechanics of Solids and Fluids. Mechanics: Analysis, 7. Sijthoff \& Noordhoff., Alphen aan den Rijn, The Netherlands; Rockville, Maryland, USA, 1980.

[28] Leonard, D. S., And Reichl, L. E. Stochastic resonance in a chemical reaction. Physical Review E 49 (Feb. 1994), 1734-1737.

[29] McNamara, B., And Wiesenfeld, K. Theory of stochastic resonance. Physical Review A (General Physics) 39 (May 1989), 4854-4869.

[30] NiCOLIS, C. Stochastic aspects of climatic transitions - responses to periodic forcing. Tellus 34 (1982), 1-9.

[31] Olver, F. W. J. Asymptotics and special functions. Computer Science and Applied Mathematics. Academic Press, a subsidiary of Harcourt Brace Jovanovich, Publishers, New York - London, 1974.

[32] Pavlyukevich, I. E. Stochastic Resonance. PhD thesis, Humboldt-Universität, Berlin, 2002.

[33] Renardy, M., And Rogers, R. C. An introduction to partial differential equations, vol. 13 of Texts in Applied Mathematics. Springer-Verlag, New York, 1993.

[34] Riani, M., and Simonotto, E. Stochastic resonance in the perceptual interpretation of ambiguous figures: A neural network model. Physical Review Letters 72 (May 1994), 3120-3123.

[35] Sellers, W. B. A global climate model based on the energy balance of the earth-atmosphere system. J. Appl. Meteor. 8 (1969), 301-320.

[36] Spano, M. L., Wun-Fogle, M., and Ditto, W. L. Experimental observation of stochastic resonance in a magnetoelastic ribbon. Physical Review A 46 (Oct. 1992), 5253-5256.

[37] Titz, S., Kuhlbrodt, T., Rahmstorf, S., and Feudel, U. On freshwaterdependent bifurcations in box models of the interhemispheric thermohaline circulation. Tellus - Series A 54, 1 (2002), 89-97. 